\title{
3. Die Erfassung des Arbeitsmarktes durch die Arbeitsverwaltung
}

\section{Registrierung der erwerbsfäbigen Bevölkerung}

Die Arbeitsverwaltung konnte in der SBZ nach dem Ende des Zweiten Weltkrieges nur in einem sehr eingeschränkten Maße auf statistische Unterlagen der Reichsanstalt für Arbeitsvermittlung und Arbeitslosenversicherung zurückgreifen, da diese im Verlauf des Krieges zu einem beträchtlichen Teil verloren gegangen waren1. Dies betraf etwa die Meldungen der Berufszählung vom 17. Mai 1939, welche nach Wirtschaftszweigen und nach Berufsgruppen aufgeschlüsselt waren. Insofern bedeutete das Kriegsende für die ostdeutschen Statistiker in zahlreichen Bereichen einen völligen Neuanfang. Die Durchführung einer Volks-, Berufs- und Betriebszählung stellte daher eine vordringliche Aufgabe für die wirtschaftlichen Zentralund Landesverwaltungen dar. Ein Sachbearbeiter im ehemaligen Statistischen Reichsamt arbeitete dazu im Sommer 1945 einen entsprechenden Entwurf aus, der am 15. August Walter Ulbricht zugeleitet wurde 2 . Darin verwies der Autor auf den „fühlbare[n] Mangel an neuen Zahlen " ${ }^{3}$ in allen vier Besatzungszonen und berief sich auf Empfehlungen der statistischen Kommission des Völkerbundes aus der Zeit vor 1933 an die Mitgliedstaaten, nach denen solche "grundlegenden“ Zählungen am 31. Dezember eines Jahrfünfts oder Jahrzehnts erfolgen sollten, um die Vergleichbarkeit der Ergebnisse gewährleisten zu können. Der Sachbearbeiter wollte diese detaillierte statistische Erhebung mit einer „allumfassenden Inventur des deutschen Volkes und der deutschen Wirtschaft in der Gegenwart und zu Beginn des Krieges" verknüpfen. Im einzelnen sollten die Bevölkerungs- und Materialverluste während des Krieges sowie die Einkommensverteilung zu Kriegsbeginn, Kriegsende und beim Stichtag der Zählung erfaßt werden. Diese Ergebnisse waren getrennt nach den einzelnen Besatzungszonen zu gliedern. Darüber hinaus wurde vorgeschlagen, die Mitglieder von NSDAP und weiteren nationalsozialistischen Organisationen zu erfassen. Der Entwurf, der eine Volks-, Berufs- und Betriebszählung am 31. Dezember 1945 vorsah, wurde sehr stark von dem pädagogischen Gedanken geleitet, einen Beitrag zur „Abwicklung des grausamen Krieges" $z u$ leisten und für die Zukunft einen erneuten Krieg zu verhindern'.

Einzelne Landesregierungen waren auch auf diesem Gebiet der ZVAS einen Schritt voraus. So erließ etwa das thüringische Landesamt für Arbeit am 9. August 1945 eine vorläufige Verordnung über die Meldung offener Stellen und die Einstellung von Arbeitskräften ${ }^{5}$. Darin wurde unter anderem die Verordnung über die Beschränkung des Arbeitsplatzwechsels vom 1. September 1939 aufgehoben. Außerdem wurde die Meldepflicht für alle privaten und öffentlichen Betriebe festgelegt. In Thüringen war diese vorläufige Verordnung eng gekoppelt mit einer Verordnung über die Registrier- und Arbeitspflicht, die am 16. August in Kraft

1 BAB, DQ 2/137, Bl. 169, DVAS-Abt. I a am 9. 4. 1948 an SMAD-Abt. Arbeitskraft.

SAPMO, NY 4182/948, Bl. 8-10.

3 Ebenda, Bl. 8.

4 Ebenda, Bl. 9.

s $\mathrm{BAB}, \mathrm{DQ} 2 / 1978$. 
trat $^{6}$. Demzufolge bestand eine Registrierpflicht für alle Männer im Alter von 14 bis 65 Jahren und alle Frauen im Alter von 14 bis 50 Jahren. Die Arbeitsämter konnten bei Verstößen gegen die Verordnung Ordnungsstrafen verhängen sowie die Lebensmittelkarten sperren lassen.

Am 15. September erschien der bereits mehrfach erwähnte SMAD-Befehl Nr. 657, der eine Vereinheitlichung für die gesamte SBZ brachte. Um eine Zählung der arbeitsfähigen Bevölkerung in den Ländern und Provinzen der SBZ nach einheitlichen Kriterien zu gewährleisten, erarbeitete die ZVAS-Abteilung für Erfassung und Einsatz der Bevölkerung einen Richtlinienkatalog, der am 24. September 1945 an die Landesarbeitsämter verschickt wurde ${ }^{8}$. Darin wurde unter anderem die Wiedereinführung des Arbeitsbuches angekündigt, das anfangs die Bezeichnung "Arbeitspaß“ trug, um vermutlich die Distanz zur nationalsozialistischen Arbeitseinsatzpolitik zu verdeutlichen. Bereits am 1. Oktober legte die ZVAS eine Verordnung über die „Meldepflicht und die Einführung eines Arbeitspasses und einer Kontrollkarte" vor, die erste ausführliche Bestimmungen enthielt ${ }^{9}$. Zeitgleich veröffentlichte die ZVAS eine Verordnung über die „Erfassung und Zählung der arbeitsfähigen deutschen Bevölkerung "10: Die Karteikarten, die von den Arbeitsämtern nach Berufs- und Altersgruppen zu sortieren und zu führen waren, sollten nach den Vorstellungen der ZVAS genaue Angaben zu jedem Beschäftigten enthalten, vor allem zum Ausbildungsgang sowie zur beruflichen Qualifikation und Weiterbildung. Nach Angaben des Landesamtes für Arbeit in Thüringen war der Erfassungsgrad unterschiedlich und primär abhängig vom jeweiligen Wirtschaftssektor. So fiel die Registrierung in die Erntezeit, was zur Folge hatte, daß die Erhebungen in der Landwirtschaft "lückenhaft" waren ${ }^{11}$. Darüber hinaus habe sich die auf dem Lande "außerordentlich hohe Zahl" von Evakuierten nur als „vorübergehend anwesend" betrachtet und daher auch nicht bei den Arbeitsämtern gemeldet. Ähnliche Schwierigkeiten ergaben sich offensichtlich auch bei der Gruppe der Selbständigen sowie den mithelfenden Familienangehörigen. Aus diesem Grunde war es wenig glaubhaft, daß das thüringische Landesamt für Arbeit den relativ geringen Anteil derjenigen, die sich der Registrierung entzogen haben, mit nur fünf Prozent angab ${ }^{12}$. Dennoch bleibt festzuhalten, daß der ZVAS bereits Anfang Oktober 1945 erste statistische Erhebungen sowie erste Arbeitsmarktberichte aus den Ländern vorlagen, die im Laufe der Zeit weiter verfeinert werden sollten.

Die Herausgabe der Verordnung über die Meldepflicht und die Einführung des "Arbeitspasses“ verzögerten sich zunächst aus nicht erkennbaren Gründen. Ver-

- Ebenda. Gleichzeitig wurde die Verordnung des Beauftragten für den Vierjahresplan zur Sicherstellung des Kräftebedarfs für Aufgaben von besonderer staatspolitischer Bedeutung vom 13.2. 1939 sowie die dazu ergangenen Anordnungen des Reichsarbeitsministeriums und des Generalbevollmächtigten für den Arbeitseinsatz aufgehoben.

7 Arbeit und Sozialfürsorge 1 (1946), S. 3.

\& $\mathrm{BAB}, \mathrm{DQ} 2 / 111, \mathrm{Bl} .8-11$.

- BAB, DQ 2/2008.

10 $\mathrm{BAB}, \mathrm{DQ} 2 / 1614$. In einer sogenannten 2. Verordnung wurden explizit dic Aufgaben der Arbeitsämter nochmals festgehalten. Beide Verordnungen sind abgedruckt in: Arbeit und Sozialfürsorge 1 (1946), S. 26-29.

" BAB, DQ 2/1978, Landesamt für Arbeit Thüringen am 2. 10. 1945 an die ZVAS, S. 3.

12 Ebenda. 
mutlich hielt die ZVAS eine rasche Veröffentlichung für nicht opportun. Am 20. Oktober 1945 erklärten Vertreter der SMAD bei einer Besprechung in Karlshorst, daß zwei getrennte Verordnungen ausgearbeitet werden sollten: „Die Verordnung über die Meldepflicht muß herausgebracht werden ohne die Verordnung über das Arbeitsbuch, die später herauszugeben ist." ${ }^{13}$ Auch bei dieser Gelegenheit machten die sowjetischen Vertreter unmißverständlich klar, daß erst nach einer Registrierung beim Arbeitsamt die Betroffenen mit der Ausgabe von Lebensmittelkarten rechnen konnten; und umgekehrt galt, daß das „Recht zum Erhalt der Lebensmittelkarten" entzogen werden konnte ${ }^{14}$. Die statistische Erfassung der arbeitsfähigen Bevölkerung wurde von der sowjetischen Besatzungsmacht des öfteren angesprochen; Karlshorst verlangte immer wieder ausführliche Berichte. Am 17. Mai 1946 erteilte der SMAD-Mitarbeiter Lamin der DVAS den Auftrag, „die Gründe für die Abwanderung der Bevölkerung nach dem Westen“ anzugeben ${ }^{15}$. Neben der Binnenwanderung, die bis zu diesem Zeitpunkt nie zur Zufriedenheit der deutschen und sowjetischen Dienststellen gesteuert werden konnte, rückte damit erstmals ein anderes Phänomen in den Blickpunkt, das ab Ende der vierziger Jahre zunehmend an Bedeutung gewinnen sollte: die Flucht in die westlichen Besatzungszonen. Die SMAD-Abteilung Arbeitskraft richtete auch in der Folgezeit kritische Nachfragen an die DVAS, die in erster Linie Einzelfragen im Zusammenhang mit den einzelnen Erhebungsbögen betrafen ${ }^{16}$. So wurden etwa die Altersaufgliederung modifiziert und zum Teil neue Berufsgruppen als Erfassungsgröße eingeführt. Offiziell galt das Junktim von Registrierung und Vergabe der Lebensmittelkarten für den gesamten Zeitraum der SBZ ${ }^{17}$, seine Bedeutung nahm jedoch sukzessive ab. Die Arbeitsämter setzten dieses Zwangsmittel immer seltener ein; der drakonische Sanktionscharakter, den die Lebensmittelkartensperre in der unmittelbaren Nachkriegszeit durchaus noch hatte, war somit weitgehend verloren gegangen.

Mitte des Jahres 1946 lag eine einheitliche betriebliche Meldekartei für die SBZ noch nicht vor. Die Arbeitsverwaltung beklagte nach wie vor die Verluste an statistischen Materialien der Reichsarbeitsverwaltung vor 1945 und ließ damit indirekt erkennen, daß dieser Rückstand immer noch nicht aufgeholt worden war ${ }^{18}$. Der Aufbau der Meldekartei sollte nach den Vorstellungen der DVAS-Leitung auf der betrieblichen Ebene erfolgen, da nur so der konkrete Arbeitskräftebedarf und das bestehende Arbeitskräfteangebot angezeigt werden konnten. Noch im Frühjahr 1949 arbeitete die HVAS Dienstanweisungen „über die Führung und Pflege der Karteien" aus ${ }^{19}$. Im Zuge der aufkommenden allgemeinen Wirtschaftsplanung stand auch eine Veränderung bei der Registrierung der Bevölkerung auf der Tagesordnung: Die Meldebögen sowie die einzelnen statistischen Kategorien sollten of-

13 BAB, DQ 2/2008, Aktenvermerk der Abt. IV, S. 1.

14 Ebenda, S. 2.

$15 \mathrm{BAB}, \mathrm{DQ} 2 / 1, \mathrm{Bl} .73$, Aktennotiz über Besprechung mit Lamin (SMAD) am 17. 5. 1946.

16 BAB, DQ 2/2040, Bl. $20 \mathrm{f}$., Aktenvermerk der DVAS-Abt. II über eine Besprechung mit der SMAD-Abt. Arbeitskraft am 30. 8. 1946.

17 So erließ beispielsweise die Landesregierung von Sachsen-Anhalt noch am 14.9. 1949 einen entsprechenden Aufruf. Vgl. BAB, DQ 2/2008.

$18 \mathrm{BAB}, \mathrm{DQ} 2 / 2064$, Denkschrift der ZVAS (Abt. II) vom 2. 7. 1946.

$19 \mathrm{BAB}, \mathrm{DQ} 2 / 2063$, Entwurf vom 29. 4. 1949. 
fensichtlich nach dem Willen einzelner Landesverwaltungen den neuen wirtschaftspolitischen Anforderungen angepaßt werden. So schlug etwa das thüringische Ministerium für Arbeit und Gesundheitswesen im Mai 1949 vor, eine „vollkommene Änderung des Erfassungssystems mit einer gleichzeitigen organisatorischen Trennung der Arbeitsverwaltung von der Sozialpolitik und der Hinwendung zur Wirtschaft" zu verbinden ${ }^{20}$.

Ein gesondertes Problem stellte die Registrierung der Ausländer und der sogenannten Staatenlosen - den Displaced Persons in der SBZ - dar. Hier forderte das sächsische Landesarbeitsamt die DVAS Anfang Dezember 1946 auf, eine entsprechende Verordnung zu erlassen, die „für dringend notwendig“ angesehen wurde ${ }^{21}$. Von dieser Regelung sollten Angehörige der alliierten Siegermächte ausgenommen werden. Die SMAD überließ es der DVAS, „nach eigenem Ermessen [zu] entscheiden", ob eine solche gesetzliche Bestimmung erforderlich war ${ }^{22}$. Eine nochmalige Rücksprache war nach Ansicht der sowjetischen Besatzungsmacht nicht mehr notwendig. Daraufhin erließ die DVAS am 16. Januar 1947 eine Verordnung, in der die oben genannte Personengruppe der Registrierpflicht unterworfen wurde ${ }^{23}$.

Damit war allerdings die Situation nicht geklärt, da die SMAD im Herbst 1947 Änderungswünsche einbrachte und damit für Verwirrung sorgte: So sollte sich die Erfassung durch die Arbeitsverwaltung nunmehr nur noch auf die Staatenlosen beschränken ${ }^{24}$. Nach den Vorschlägen aus Karlshorst hatten sich Ausländer bei den Arbeitsämtern nicht zu melden; nur bei Arbeitsaufnahme sollten Arbeitsbücher ausgegeben werden. Der Grund für das sowjetische Eingreifen lag darin, daß der Kontrollratsbefehl Nr. 3 eine Verpflichtung von Ausländern zur Registrierung bei den Arbeitsämtern nicht vorsah ${ }^{25}$. Auf einer Arbeitstagung in Leipzig am 6./7. Januar 1948 betonten Vertreter der DVAS, daß die „Verhältnisse [...] inzwischen jedoch unhaltbar geworden [seien], da erfahrungsgemäß eine ganze Anzahl von Ausländern den Schwarzmarkt bevölkern" würden ${ }^{26}$. Verhandlungen mit der SMAD waren erneut aufgenommen worden. Nach Mitteilung der Berliner Zentralverwaltung mußte die Verordnung vom 16. Januar 1947 aufgehoben werden, da sich „einzelne Staaten dagegen auf[ge]lehnt" hätten. Die einzelnen Landesregierungen reagierten offenbar unterschiedlich: Während in Mecklenburg-Vorpommern die Registrierpflicht für Ausländer weiterhin galt, hatte das Berliner Amt für Arbeit der Kommandantur eine entsprechende Vorlage unterbreitet, über die aber noch weiter verhandelt werden mußte. Die DVAS übte sich in Zurück-

20 ThHStA, Land Thüringen, Ministerium für Wirtschaft und Arbeit, Bd. 3597, Bl. 192-194, hier Bl. 194, Studzinski am 17. 5. 1949 an die HVAS.

21 BAB, DQ 2/2064, Aktenvermerk der DVAS-Abt. I über eine Besprechung am 6./7. 12. 1946.

$22 \mathrm{BAB}, \mathrm{DQ} 2 / 1, \mathrm{Bl}$. 27, Aktenvermerk über die Besprechung mit der SMAD in Karlshorst am 14.1. 1947.

23 Arbeit und Sozialfürsorge 2 (1947), S. 2.

24 $\mathrm{BAB}, \mathrm{DQ} 2 / 2040, \mathrm{Bl}$. 94, Niederschrift der Abt. I b über eine Besprechung in Karlshorst am 18. 9. 1947. Eine entsprechende Verordnung mußte die DVAS am 10.7.1947 erlassen. Vgl. Arbeit und Sozialfürsorge 2 (1947), S. 328.

$25 \mathrm{BAB}, \mathrm{DQ} 2 / 1711$, Niederschrift der DVAS-Abt. I b über Besprechung mit der SMAD in Karlshorst am 23. 12. 1947.

$26 \mathrm{BAB}, \mathrm{DQ} 2 / 1537$, Niederschrift der Arbeitstagung, S. 24. 
haltung und machte nur deutlich, daß sie dem Vorgehen der mecklenburgischen Landesverwaltung nicht zustimmen konnte.

Die DVAS erließ am 25. März 1948 eine Verordnung, die den Kontrollratsbefehl völlig neu interpretierte. $\mathrm{Zu}$ den registrierpflichtigen Personen gehörten nun doch „alle Personen ausländischer Staatsangehörigkeit oder staatenlose Personen, die in der sowjetischen Besatzungszone ihren Wohnsitz" hatten ${ }^{27}$. Ausgenommen blieben Staatsangehörige der alliierten Besatzungsmächte, Mitglieder von diplomatischen oder Militärmissionen sowie von internationalen Organisationen. Auffallend ist der Umstand, daß diese Verordnung kurz nach dem Auszug des sowjetischen Vertreters aus dem Alliierten Kontrollrat veröffentlicht wurde. Das läßt zumindest die Vermutung zu, daß die SMAD zuvor in dieser Angelegenheit nicht vorpreschen und den drei westlichen Siegermächten keinen Vorwand liefern wollte, die Gespräche abzubrechen. Insbesondere die Länder fühlten sich jedoch bei der Ausarbeitung dieser Verordnung übergangen ${ }^{28}$.

Aus der Registrierpflicht für ausländische Staatsbürger ließ sich keineswegs das Recht der Arbeitsämter zur Arbeitseinweisung ableiten. Da die Verordnung vom 25. März 1947 diese Frage jedoch unbeantwortet ließ, nahmen vor allem einige Bürgermeister eine eigenmächtige Gesetzesauslegung vor und veranlaßten des öfteren die Einweisung in Großbetriebe. So hatte sich beispielsweise ein in Brandenburg lebender schweizerischer Staatsbürger an die dortige Landesregierung gewandt, nachdem er den Einweisungsbefehl in das Stahl- und Walzwerk Hennigsdorf erhalten hatte ${ }^{29}$. Unterstützt wurde der Betroffene von der schweizerischen Heimschaffungsdelegation, die ausdrücklich darauf verwies, daß aufgrund einer zwischenstaatlichen Vereinbarung zwischen der Schweiz und Deutschland, die noch vor Ende des Zweiten Weltkrieges geschlossen worden war, Schweizer in Deutschland nicht den Arbeitseinweisungen Folge zu leisten brauchten ${ }^{30}$. Das Landesarbeitsamt in Potsdam hob daraufhin die Arbeitseinweisung auf und stellte gleichzeitig bei der HVAS den Antrag, eine grundsätzliche Klärung herbeizuführen. Die HVAS ließ sich sehr viel Zeit und informierte relativ spät die Deutsche Verwaltung des Innern über den Vorgang und bat um Mitteilung, ob mit anderen Staaten ähnliche Abkommen vorlägen ${ }^{31}$. Ein Antwortschreiben ist nicht mehr auffindbar. Vermutlich sah die Arbeitsverwaltung von einer Einweisung dieser zahlenmäßig ohnehin kleinen Gruppe ab; zumindest sind weitere Fälle nicht mehr aktenkundig geworden.

\section{Wiedereinfübrung des Arbeitsbuches}

Ein zentrales Instrument zur Erfassung und Steuerung des Arbeitskräftepotentials stellte in der SBZ/DDR das Arbeitsbuch dar; in ihm wurden berufliche Qualifikation und beruflicher Werdegang jedes Beschäftigten detailliert wiedergegeben.

27 Arbeit und Sozialfürsorge 3 (1948), S. $137 \mathrm{f}$.

28 So äußerte sich etwa der Vertreter des mecklenburgischen Ministeriums für Arbeit und Sozialfürsorge Rosenträger: $\mathrm{BAB}, \mathrm{DQ} 2 / 1537$, Niederschrift über den Verlauf der am 27./28. 4. 1948 stattgefundenen Arbeitstagung der HVAS (Abt. I b) in Erfurt, S. 12.

$29 \mathrm{BAB}, \mathrm{DQ} 2 / 262, \mathrm{Bl}$. 81, Landesarbeitsamt Potsdam am 15. 7. 1948 an die HVAS.

30 Ebenda, Bl. 82, Schweizerische Heimschaffungsdelegation am 5.7.1948 an Rudolf $H$.

31 Ebenda, Bl. 91, HVAS (Herm) am 19. 5. 1949 an DVdI. 
In der SBZ wurde frühzeitig die Einführung des Arbeitsbuches diskutiert. Während die sowjetische Besatzungsmacht diesen Schritt befürwortete und auch erwartete, blieb die DVAS zunächst noch skeptisch, da hierbei auf ein Element staatlicher Arbeitsmarktpolitik zurückgegriffen wurde, das bereits vom NS-Regime eingesetzt worden war und das daher diskreditiert schien ${ }^{32}$. Die deutsche Arbeitsverwaltung fürchtete letztlich einen Ansehensverlust und schob eine Entscheidung hinaus.

Bereits am 8. September 1945 erteilte die SMAD-Abteilung Arbeitskraft der ZVAS den Auftrag, „baldmöglichst“ einen einheitlichen „Arbeitspaß“ einzuführen, der die Voraussetzung für die Vergabe von Lebensmitteln sein sollte ${ }^{33}$. Die Abteilung IV (Erfassung und Arbeitseinsatz der Bevölkerung) legte rund drei Wochen später einen ersten Verordnungsentwurf vor, der die Meldepflicht mit der Vergabe von "Arbeitspässen“ verband ${ }^{34}$. Demzufolge hatten alle Beschäftigten im Besitz eines "Arbeitspasses“ zu sein, in dem der Arbeitgeber Beginn und Funktion der jeweiligen Tätigkeit eintragen sollte. Die Arbeitsämter hatten ihrerseits eine Kartei über die Arbeitspässe zu führen. Karlshorst sah keinerlei Probleme für eine rasche Veröffentlichung der Verordnung; die zuständige Abteilung Arbeitskraft ging davon aus, daß noch Ende des Jahres 1945 mit dem Druck der mittlerweile umbenannten Arbeitsbücher begonnen werden konnte ${ }^{35}$. Die Bereitstellung entsprechender Papierkontingente war von der SMAD offenbar genehmigt worden. Angeblich waren sich wohl auch die vier Siegermächte im Alliierten Kontrollrat über die Herausgabe eines einheitlichen Arbeitsbuches für ganz Deutschland einig ${ }^{36}$. Einer raschen Verabschiedung schien nichts im Wege zu stehen, und auch der FDGB signalisierte seine prinzipielle Zustimmung 37 . In inhaltlichen Fragen herrschte Übereinstimmung zwischen den sowjetischen und den deutschen Dienststellen: Auch die ZVAS hielt die Wiedereinführung eines Arbeitsbuches „für das Wirtschaftsleben unbedingt erforderlich" und bezog diese Feststellung auf alle vier Besatzungszonen unter Berufung auf die Bestimmung des Potsdamer Abkommens, nach der Deutschland als wirtschaftliche Einheit zu betrachten war ${ }^{38}$. Neben der Forderung nach einer Regelung für alle vier Besatzungszonen sprach sich die ZVAS-Abteilung für eine Ausdehnung der Arbeitsbuchpflicht auf die gesamte arbeitnehmende Bevölkerung aus. Ende November stimmte die SMAD dem deutschen Vorschlag zu, den vorliegenden Verordnungsentwurf den

32 Obwohl das Arbeitsbuch 1935 eingeführt worden war, zog sich die Durchsetzung doch bis zum Frühjahr 1939 hin. Erst zu diesem Zeitpunkt galt die Registrierung der Bevölkerung als abgeschlossen. Befreit von der Arbeitsbuchpflicht waren Beamte, Teile der Selbständigen sowie die freien Berufe. Vgl. Mason, Sozialpolitik im Dritten Reich, S. 162.

$33 \mathrm{BAB}, \mathrm{DQ} 2 / 2040, \mathrm{Bl}$. 1. Auch der SMAD-Befehl Nr. 65 vom 15. 9. 1945 sah die Einführung von Arbeitsbüchern für die abhängig Beschäftigten in einzelnen zentralen Wirtschaftsbereichen sowie Registrierkarten für die Arbeitslosen vor. Vgl. Arbeit und Sozialfürsorge 1 (1946), S. 3.

34 $\mathrm{BAB}, \mathrm{DQ} 2 / 2483$, Entwurf zu einer Verordnung über die Meldepflicht und die Einführung eines Arbeitspasses und einer Kontrollkarte vom 1.10. 1945.

35 BAB, DQ 2/2006, Aktenvermerk über eine Besprechung mit Remissow am 22.11. 1945.

36 Ebenda.

37 BAB, DQ 2/2069, Roman Chwalek (FDGB) am 26.11. 1945 an Gustav Gundelach (ZVAS).

$38 \mathrm{BAB}, \mathrm{DQ}$ 2/2006, Memorandum der ZVAS-Abt. II vom 27. 11. 1945, S. 1. 
Landes- und Provinzialverwaltungen zuzuleiten, „damit es [das Arbeitsbuch] möglichst bald zur Anwendung gelangt" ${ }^{\text {"39. }}$.

Wenige Wochen später ergaben sich unerwartete Verzögerungen, da die sowjetische Besatzungsmacht damit liebäugelte, eine Ersatzkarte und kein Arbeitsbuch einzuführen ${ }^{40}$. Inwieweit übergeordnete Interessen, vor allem deutschlandpolitische Absichten dahintersteckten, kann nur vermutet werden. Auffallend ist jedoch, daß die SMAD auch hier mit einem Mal als Bremser auftrat und von einem Vorhaben, das sie zunächst initiiert und nachdrücklich unterstützt hatte, wieder etwas Abstand nahm. Die ZVAS meldete dagegen „erhebliche Bedenken“41 an und verwies darauf, daß die Ersatzkarte zwar Aufschluß über die verschiedenenen Beschäftigungsverhältnisse geben würde; sie enthalte aber keine Angaben über die berufliche Ausbildung. Beides sei für die Arbeitsverwaltung aber eine "grundlegende Voraussetzung, um den Arbeitseinsatz der Bevölkerung zweckmäßig und rationell lenken zu können." Die Ersatzkarte - so die Schlußfolgerung der Berliner Zentralverwaltung - könne nur als "Notbehelf“ eingesetzt werden und sei als „verbindliches Dokument [...] gänzlich abzulehnen." Für die sowjetische Besatzungsmacht genoß zu diesem Zeitpunkt das Ziel einer Einigung mit den drei Westmächten im Alliierten Kontrollrat Priorität, und das war wohl auch der eigentliche Grund für den Vorschlag, eine provisorische Lösung mit der Ersatzkarte zu favorisieren. Gegenüber Vertretern der ZVAS gab der SMAD-Mitarbeiter Remissow am 22. Januar 1946 bekannt, daß im Kontrollrat eine Einigung über die Herausgabe eines einheitlichen Arbeitsbuches erzielt worden sei ${ }^{42}$.

Der Berliner Magistrat hatte auch bei der Wiedereinführung des Arbeitsbuches eine Vorreiterrolle eingenommen; in der früheren Reichshauptstadt lag bereits am 26. Juni 1945 eine Verordnung vor ${ }^{43}$. Der für den Arbeitseinsatz zuständige Dezernent meldete der ZVAS, daß der Alliierte Kontrollrat eine einheitliche Lösung anstrebe und deshalb eine vorschnelle Entscheidung in der SBZ zu vermeiden $\operatorname{sei}^{44}$. Die ZVAS-Führung schien sich langsam mit diesem Gedanken anzufreunden, zumal sie ähnliche Signale auch von der SMAD-Abteilung Arbeitskraft empfangen hatte. Die Verhandlungen im Kontrollrat waren dann Ende Januar ins Stocken geraten, und die zuständigen SMAD-Vertreter in Karlshorst deuteten gegenüber der Berliner Zentralverwaltung an, daß nun doch das Arbeitsbuch, „so wie es im Entwurf [der ZVAS] vorliege, sofort heraus[gebracht]" werden solle45.

Für die SMAD und ZVAS stellte das Arbeitsbuch eine wichtige Voraussetzung für eine zielgerichtete Arbeitskräftelenkung dar; daran bestand kein Zweifel. Allein die Frage des Zeitpunktes blieb strittig und konnte letztlich nur von der sowjetischen Besatzungsmacht entschieden werden. Auch der FDGB-Bundesvorstand äußerte sich grundsätzlich zustimmend zu der geplanten Maßnahme, allerdings mit einer nicht unerheblichen Abweichung: So vertrat der FDGB den

39 $\mathrm{BAB}, \mathrm{DQ} 2 / 1, \mathrm{Bl} .15$, Aktenvermerk über Besprechung mit Remissow am 28. 11. 1945.

40 Dies geht aus einem Schreiben der ZVAS-Abt. II b vom 16. 1. 1946 an die SMAD hervor, in: BAB, DQ 2/2006.

+1 Ebenda.

42 BAB, DQ 2/1, Bl. 17, Aktenvermerk Gundelachs.

43 Vgl. BAB, DQ 2/2069, Aktenvermerk der ZVAS vom 31. 1. 1946, S. 1.

44 Ebenda.

45 BAB, DQ 2/1, Bl. 37 f., Aktenvermerk über Besprechung mit Remissow am 13. 2. 1946. 
Standpunkt, daß das Arbeitsbuch „bei der nächsten Gelegenheit abgeschafft werden müsse" ${ }^{46}$. Zum gegenwärtigen Zeitpunkt könne aber - so die pragmatische Stellungnahme von seiten der Gewerkschaften - nicht darauf verzichtet werden.

Während die SMAD mit der DVAS bereits die praktische Durchführung erörterte und am 3. September 1946 vorschlug, die Drucklegung der Arbeitsbücher den Landes- bzw. Provinzialämtern zu überlassen ${ }^{47}$, mußten die zuständigen Abteilungen innerhalb der ZVAS erst noch den endgültigen Verordnungsentwurf weiter ausarbeiten. Im Laufe des Septembers lag ein überarbeiteter Entwurf der Abteilung II vor, der sich nur noch auf das Arbeitsbuch und die Kontrollkarte beschränkte ${ }^{48}$; dagegen waren Bestimmungen über die Registrierung herausgelöst worden. Arbeitsbuchpflichtig waren nunmehr alle unselbständig Beschäftigten, allerdings mit einigen Ausnahmen: geringfügig Beschäftigte, Mitglieder der Besatzungen von "Seefahrzeugen" (diese führten ein Seefahrtbuch), Heimarbeiter und Hausgewerbetreibende sowie die Gruppe der mithelfenden Familienangehörigen. Der Verordnungsentwurf legte außerdem fest, daß der Arbeitnehmer sein Arbeitsbuch bei Aufnahme der Tätigkeit „unverzüglich dem Arbeitgeber zur Vornahme der notwendigen Eintragungen vorzulegen" hatte ${ }^{49}$. Die Eintragungen des Arbeitgebers mußten anschließend vom Arbeitsamt mit einem Sichtvermerk bestätigt werden. Wohnortwechsel, Namensänderung und vor allem Arbeitsplatzwechsel waren ebenfalls dem Arbeitsamt sofort mitzuteilen, das zudem der Beendigung eines Arbeitsverhältnisses zustimmen mußte. Schwarzarbeit sollte dadurch verhindert werden, daß den Arbeitgebern die Anstellung von Personen, die nicht über ein Arbeitsbuch verfügten, untersagt wurde. Dadurch sollte die Position der Arbeitsämter insgesamt gestärkt werden. Für den Fall, daß ein Arbeitsbuchpflichtiger nach Auflösung eines Arbeitsvertrages nicht sofort in eine andere Arbeit vermittelt werden konnte, sah der Entwurf den Einzug des Arbeitsbuches durch das Arbeitsamt vor, das dem Betroffenen dann eine Kontrollkarte für die Dauer der Arbeitslosigkeit aushändigte ${ }^{50}$. Darüber hinaus wurde festgelegt, daß das Arbeitsbuch im Besitz der Beschäftigten zu bleiben hatte. Ausführliche Strafbestimmungen (Geld- und Gefängnisstrafen) untermauerten den festen Entschluß der DVAS, die Verordnung kompromißlos umzusetzen und das Arbeitsbuch in der SBZ rasch einzuführen.

Die Juristische Abteilung setzte sich eingehend mit dem Verordnungsentwurf auseinander und arbeitete eine Liste mit Verbesserungsvorschlägen aus, die sich im wesentlichen auf inhaltliche Präzisierungen und sprachliche Veränderungen bezogen $^{51}$. Darüber hinaus sprach sie sich für eine Vergrößerung des Personenkreises aus, welcher der Arbeitsbuchpflicht unterliegen sollte: Nicht nur die unselbständig, sondern auch die selbständig Beschäftigten sollten nach den Vorstellungen der Juristischen Abteilung erfaßt werden. Aufschlußreich war außerdem

46 BAB, DQ 2/1237, Bericht der DVAS (Jurist. Abt.) über die Tagung der arbeitsrechtlichen Kommission des FDGB am 9./10. 5. 1946.

$47 \mathrm{BAB}, \mathrm{DQ} 2 / 2040, \mathrm{Bl}$. 21, Aktenvermerk der DVAS (Abt. II) über Besprechung mit der SMADAbteilung Arbeitskraft am 30. 8. 1946.

48 BAB, DQ 2/2483.

49 Ebenda, $\$ 12$.

so Ebenda, $\$ 15$ und 16.

$51 \mathrm{BAB}, \mathrm{DQ} 2 / 81, \mathrm{Bl}$. 143 f., Juristische Abt. am 2. 10.1946 an die Abt. II. 
die Bemerkung, daß „die ganze Diktion des Entwurfes viel zu sehr den Charakter eines unter der Naziherrschaft erlassenen Gesetzes trägt, von der wir uns künftig freihalten sollten" 52 . Daher kündigte die Juristische Abteilung, die eine verständliche "Gesetzessprache" anmahnte, auch die Ausarbeitung eines Gegenentwurfes $\mathrm{an}^{53}$. Beide Abteilungen konnten sich in der Folgezeit darauf verständigen, einen neuen Entwurf „gemeinschaftlich" auszuarbeiten ${ }^{54}$, um nicht zu sehr in zeitlichen Verzug zu geraten. Vergleichsweise unbedenklich fanden dagegen beide DVASAbteilungen die im Entwurf vorgesehenen Strafbestimmungen, die starke Ähnlichkeiten zur Verordnung von 1939 aufwiesen ${ }^{55}$. Etwa zeitgleich nahm die DVAS Gespräche mit den Landesarbeitsämtern auf, um die konkrete Umsetzung der Verordnung abzustimmen. Dabei standen vor allem die Papierzuteilung, Drucklegung und Auslieferung der Arbeitsbücher in den Ländern im Mittelpunkt ${ }^{56}$. Angesichts der unzureichenden Versorgung mit Papier und des Bewirtschaftungssystems, das häufigen Änderungen durch die sowjetische Besatzungsmacht unterworfen war, mußte mit erheblichen Verzögerungen gerechnet werden. Eine flächendeckende und rasche Einführung der Arbeitsbücher schien gefährdet zu sein. Noch Ende September 1947 berichtete das sächsische Landesarbeitsamt über einen gravierenden Papiermangel; infolgedessen konnte dort mit dem Neudruck der Arbeitsbücher erst im Verlauf des vierten Quartals begonnen werden ${ }^{57}$.

Die endgültige Fassung der Verordnung über die Wiedereinführung eines Arbeitsbuches und die Einführung einer Kontrollkarte, die zuvor noch von der SMAD genehmigt worden war und die schließlich am 15. Februar 1947 in Kraft treten konnte, enthielt die wichtigsten Verbesserungsvorschläge der Juristischen Abteilung ${ }^{58}$. Vor allem war die Arbeitsbuchpflicht erheblich ausgeweitet worden; von der Verordnung ausgenommen blieben nur noch die geringfügig Beschäftigten sowie volksschulpflichtige Kinder. Dagegen wurden die Heimarbeiter und Hausgewerbetreibenden zu der Gruppe der unselbständig Beschäftigten hinzugezählt, die somit ein Arbeitsbuch führen mußten ${ }^{59}$. Arbeitsbuchpflichtig wurden nunmehr auch die mithelfenden Familienangehörigen mit Ausnahme der Ehefrauen und der minderjährigen Kinder. Anfang Januar 1948 machte die DVAS die Landesämter darauf aufmerksam, daß weitere kleinere Personengruppen von der Arbeitsbuchpflicht befreit blieben: So etwa die freischaffenden Künstler, die Geistlichen sowie die Angehörigen der Ordensgemeinschaften ${ }^{60}$. Da die Geistlichen und Ordensangehörigen durch den SMAD-Befehl Nr. 153 vom 29. Novem-

52 Ebenda, Bl. 142, Juristische Abteilung am 4. 10. 1946 an Abt. II.

53 Ebenda.

${ }^{54}$ BAB, DQ 2/81, Bl. 210, Vermerk der Juristischen Abt. vom 6.11. 1946 für Vizepräsident Voß (Abt. II).

55 Vgl. ebenda, Bl. 282, Juristische Abt. am 22.11. 1946 an das Provinzialamt für Arbeit und Sozialfürsorge in Halle.

56 BAB, DQ 2/2064, Aktenvermerk der DVAS-Abt. J über Besprechung mit dem LAA Sachsen am 6./7. 12. 1946, S. 2.

57 BAB, DQ 2/1537, Niederschrift über den Verlauf der Arbeitstagung in Neuruppin (25.-27. 9. 1947), S. 15.

58 Arbeit und Sozialfürsorge 2 (1947), S. 38-42.

39 Ebenda, $\$ 4$.

so BAB, DQ 2/1537, Niederschrift über den Verlauf der Arbeitstagung in Leipzig am 6./7. 1. 1948, S. 32 . 
ber $1945^{61}$ von der Melde- und Arbeitseinsatzpflicht nicht ausdrücklich befreit worden waren, schien der Juristischen Abteilung eine einheitliche gesetzliche Regelung für die gesamte SBZ geboten zu sein ${ }^{62}$.

Für zahlreiche Betriebe erwies sich die Bestimmung, daß das Arbeitsbuch beim Beschäftigten verblieb und für jede Änderung von der betrieblichen Verwaltung immer wieder angefordert werden mußte, als nicht sehr praktikabel. So sprachen sich etwa die Buna-Werke aber auch das zuständige Landesamt in Halle für den Verbleib der Arbeitsbücher bei den Betrieben aus ${ }^{63}$. Dies diene - so die Befürworter - den Betriebsüberprüfungen, da sofort der „berufsrichtige“ Einsatz kontrolliert werden könne. Darüber hinaus ließe sich der Verschleiß an Büchern reduzieren. Die DVAS nahm eine gegenteilige Position ein und verteidigte die Verordnungsbestimmung mit dem Hinweis, daß das Arbeitsbuch den Charakter eines Ausweises besitze und sich daher bei der Einstufung in die Lebensmittelkartengruppen und sogar bei Straßenkontrollen als nützlich erweisen würde. Vor einer abschließenden Entscheidung wollte die DVAS die Stellungnahmen der einzelnen Landesämter nochmals einholen. Diese stimmten mehrheitlich für die bestehende Regelung, d.h. für die Aushändigung der Arbeitsbücher an die Arbeitnehmer. Auf der Leipziger Arbeitstagung am 6./7. Januar 1948 wurde daher der Antrag des Landesamtes für Arbeit in Halle abgelehnt ${ }^{64}$. Auch die SMAD-Abteilung Arbeitskraft sprach sich in der Folgezeit für einen Verbleib der Arbeitsbücher bei den Betrieben aus, wollte diese Position, die wohl für die sowjetische Besatzungsmacht nur von untergeordneter Bedeutung war, aber nicht gegenüber der HVAS durchsetzen ${ }^{65}$. Somit blieb es weiterhin bei der bestehenden Regelung.

Während die Landesregierungen an der erlassenen Verordnung keine inhaltliche Kritik übten, beanstandeten sie doch den eingeschlagenen Verfahrensweg, bei dem sie weitgehend ausgeschlossen worden waren. Das Justizministerium von Sachsen-Anhalt äußerte Bedenken, "weshalb derartig wichtige Bestimmungen nicht als Gesetz vom Landtage beschlossen“ wurden ${ }^{66}$. Zu diesem Zeitpunkt sahen die Länder das Recht der DVAS, bindende Verordnungen für die SBZ zu erlassen, immer noch als umstritten an. Die Berliner Zentralverwaltung wischte diese rechtlichen Bedenken, hinter denen eigene landespolitische Interessen standen, beiseite. Abteilungsleiter Kreil betonte in seinem Antwortschreiben, daß aus "rein sachlichen und technischen Gründen" es nicht möglich sei, die Wiedereinführung des Arbeitsbuches der Ländergesetzgebung zu überlassen ${ }^{67}$. Der DVASVertreter ging darüber hinaus auch auf die prinzipielle Frage des Verhältnisses zwischen Zentral- und Landesverwaltung ein, die er eindeutig beantwortete: Es

6) Arbeit und Sozialfürsorge 1 (1946), S. 3 f.

62 BAB, DQ 2/2483, Juristische Abt. (Holling) am 27. 8. 1948 an Abt. I.

63 LA Magdeburg LHA, Rep. K MW, Nr. 10148, Bl. 66, Bericht über Arbeitstagung in Neuruppin am 25./26. 7. 1947.

64 SAPMO, DY 34/20149, Bericht der FDGB-HA 3. Ein entsprechender Beschluß, der auf der Arbeitstagung gefaßt wurde, findet sich im ausführlichen Verlaufsprotokoll, S. 11, in: BAB, DQ $2 / 1537$.

$65 \mathrm{BAB}, \mathrm{DQ}$ 2/2483, Aktenvermerk Grodeckis über Besprechung in Karlshorst am 30. 3. 1948.

66 $\mathrm{BAB}, \mathrm{DQ} 2 / 2069$, Justizministerium von Sachsen-Anhalt am 11.10.1947 an das Ministerium für Arbeit und Sozialpolitik in Halle.

67 Ebenda, Leiter der DVAS-Abt. I b am 4. 11. 1947 an das Ministerium für Arbeit und Sozialpoltik von Sachsen-Anhalt. 
sei unbegründet, „daß zu einer Zeit, in der Zentralverwaltungen für ganz Deutschland verlangt werden, ausgerechnet aus einem Land unserer Zone einer bestehenden Zentralverwaltung das Recht abgesprochen werden soll, wenigstens im sowjetischen Besatzungsgebiet eine Vereinheitlichung der Gesetzgebung herbeizuführen, in Fragen, die nur einheitlich gelöst werden können.“

Im Sommer 1948 war die Verteilung der neu gedruckten Arbeitsbücher an die Beschäftigten keineswegs abgeschlossen. Die SMAD drängte mehrmals auf einen zügigen Abschluß und äußerte gegenüber Vertretern der DVAS sogar den Verdacht, „die Ausstellung würde [...] leichtfertig oder zumindest nachlässig behandelt werden" 68 . Die DVAS, die sich in dieser Angelegenheit stets unter Rechtfertigungsdruck sah, verwies auf die Schwierigkeiten bei der Papier- und Kartonbeschaffung, die letztlich die Auslieferung der Arbeitsbücher verzögert habe. Der SMAD-Vertreter Iljuschin machte am 1. Juli unmißverständlich klar, daß die Fertigstellung "endlich“ abzuschließen sei; dazu sollte auch die DWK eingeschaltet werden, um die Lieferung von Papier sicherzustellen ${ }^{69}$. Mecklenburg-Vorpommern bildete unter den Ländern der SBZ das Schlußlicht und mußte Anfang 1949 der HVAS die Mitteilung machen, daß das Arbeitsbuch „bisher noch nicht herausgebracht werden konnte“, was primär mit der schlechten finanziellen Ausstattung im Landeshaushalt zusammenhing ${ }^{70}$. Das brachte dem mecklenburgischen Minister für Sozialwesen Friedrich Burmeister heftige Vorhaltungen von seiten der SMAD 71 und der $\mathrm{HVAS}^{72}$ ein. Sehr weit fortgeschritten waren dagegen die Landesämter in Sachsen und Thüringen; hier stand die Ausgabe der Arbeitsbücher kurz vor dem Abschluß ${ }^{73}$. Verzögerungen meldete offensichtlich die brandenburgische Landesverwaltung, und in Sachsen-Anhalt sollte die Verteilung erst Anfang März 1949 erfolgen ${ }^{74}$.

Die sowjetische Besatzungsmacht intervenierte nur selten. Da mit der DVAS Konsens darüber herrschte, daß das Arbeitsbuch wiedereingeführt werden sollte und dafür eine Verordnung auszuarbeiten war, war das nicht weiter verwunderlich. Bei der praktischen Durchführung schaltete sich die SMAD sehr wohl ein, darauf ist bereits hingewiesen worden. Dagegen blieb die inhaltliche Ausarbeitung der Verordnung eine Angelegenheit der zuständigen Zentralverwaltung in Berlin. Vertreter der Abteilung Arbeitskraft brachten zwar des öfteren eigene Vorschläge ein, die offenbar aber keinen verbindlichen Charakter hatten. So schlug etwa Iljuschin am 30. März 1949 vor, im Arbeitsbuch eine neue Rubrik aufzunehmen, „in welcher die Gründe der Entlassungen, z. B. Diebstahl, Nichteignung usw. eingetragen“ werden sollten ${ }^{75}$. Die HVAS riet "dringend" davon ab und verwies auf die geltende Verordnung, die solche Eintragungen ausdrücklich untersagte.

68 BAB, DQ 2/2040, Bl. 129, Niederschrift über die am 22. 5. 1948 stattgefundene Besprechung bei der SMAD.

69 Ebenda, Bl. 143, Aktennotiz über Besprechung bei SMAD am 1. 7. 1948.

70 BAB, DQ 2/2063, Ministerium für Sozialwesen von Mecklenburg-Vorpommern am 28.1.1949 an die HVAS.

7 BAB, DQ 2/2049, Bl. 22, Protokoll der Abteilungsleitersitzung am 7. 2. 1949.

$72 \mathrm{BAB}, \mathrm{DQ} 2 / 2063$, Litke am 9. 4. 1949 an Burmeister.

73 Ebenda, Aktenvermerk Donaus (Abt. I a) vom 11.2. 1949.

74 Ebenda.

75 BAB, DQ 2/2483, Aktenvermerk Grodeckis über Besprechung in Karlshorst am 30. 3. 1949. 
Um ein zügiges und vor allem ein einheitliches Arbeitsverfahren bei den Arbeitsämtern zu gewährleisten, hatte die HVAS etwas verspätet im Sommer 1948 damit begonnen, eine entsprechende Dienstanweisung auszuarbeiten ${ }^{76}$. Sie unterrichtete die Landesämter für Arbeit über das Vorhaben auf den unregelmäßig einberufenen Arbeitstagungen. Konflikte ergaben sich hier jedoch nicht mehr77, so daß die Dienstanweisung über die Ausfertigung, Schließung und Wiedereröffnung von Arbeitsbüchern in der Dezemberausgabe der Zeitschrift „Arbeit und Sozialfürsorge" veröffentlicht werden konnte ${ }^{78}$.

Eine Änderung der Arbeitsbuchverordnung wollte die HVAS im Sommer 1949 durch das Sekretariat der DWK vornehmen lassen. Ausgangspunkt waren die beiden Paragraphen 1 und 14 der Verordnung über die Wiedereinführung eines Arbeitsbuches, welche die Arbeitsämter verpflichteten, die Arbeitsbücher der nicht sofort zu vermittelnden Erwerbslosen einzuziehen. Aus den Tätigkeitsberichten der Arbeitsämter ging hervor, daß dadurch eine unnötige Mehrbelastung auftrat. Darüber hinaus waren zahlreiche Ämter nicht in der Lage, „die ihnen zur Verwahrung übergebenen Arbeitsbücher unter sicherem Verschluß zu halten"79. Die HVAS schloß sich diesen Bedenken an und plädierte für eine Nachbesserung der Gesetzeslage, die darauf hinauslaufen sollte, die Arbeitsbücher im Besitz der Erwerbslosen zu belassen ${ }^{80}$. Der Leiter des Sekretariatsbüros der DWK, Erwin Lampka, hatte zunächst keinerlei Einwände gegen den eingebrachten Entwurf81. Überraschenderweise lehnte das Sekretariat der DWK auf seiner Sitzung am 27. Juli die Vorlage jedoch ab, was weniger inhaltliche als vielmehr formale Gründe hatte. Gleichzeitig wurde nämlich der HVAS der Auftrag erteilt, eine Anweisung "entsprechend der Vorlage" an die Landesarbeitsämter herauszugeben ${ }^{82}$. Deren Ausarbeitung zog sich bis Anfang Januar 1950 hin; erst zu diesem Zeitpunkt wurden die Arbeitsämter von der Verpflichtung entbunden, Arbeitsbücher von Erwerbslosen während des Zeitraumes der Arbeitslosigkeit einzuziehen und aufzubewahren ${ }^{83}$.

\section{Gesetz zur Bekämpfung von Scheinselbständigkeit}

Grundlage einer effektiven Arbeitskräftelenkung bildete für die Arbeitsverwaltung die vollständige Registrierung der erwerbsfähigen Bevölkerung. Frühzeitig glaubte die ZVAS dem Bestreben von Teilen der Bevölkerung entgegentreten zu müssen, die sich dieser Erfassung durch den Gang in die berufliche Selbständigkeit angeblich entziehen wollten. Dahinter stand das Problem der von den Arbeitsämtern nicht vermittelten und nicht registrierten Arbeitskräfte - ein Phäno-

76 BAB, DQ 2/2047, Entwurf der HVAS-Abt. I b vom 19. 7. 1948.

77 Vgl. BAB, DQ 2/1762, Niederschrift über den Verlauf der Arbeitstagung in Bad Kösen am 5./6. 10. 1948, S. 14.

78 Arbeit und Sozialfürsorge 3 (1948), S. 451-453.

$79 \mathrm{BAB}, \mathrm{DQ} 2 / 2063$, Notiz Bracks vom 30.5. 1949 an das Sekretariat der DWK.

so Einen Anordnungsentwurf legte die HVAS (Abt. I a) am 7.6. 1949 vor, in: BAB, DQ 2/2063.

81 BAB, DC 15/II/1-37, Bl. 46, Vorlage vom 30.6. 1949 für die Sekretariatssitzung. Die Vorlage war von Lampka unterzeichnet worden.

$82 \mathrm{BAB}, \mathrm{DC} 15 / 412, \mathrm{Bl} .2$.

$83 \mathrm{BAB}, \mathrm{DQ} 2 / 903, \mathrm{Bl} .175$, Begründung Litkes (Ministerium für Arbeit und Gesundheitswesen) vom 6.1.1950. 
men, das signifikant für diesen Zeitraum bis ungefähr 1948 war und im übrigen in allen vier Besatzungszonen zu beobachten war. Unter dem Schlagwort der „Schwarzmarktzeit" verbergen sich diese Entwicklungen, die von der staatlichen Verwaltung frühzeitig erkannt worden waren, denen aber nur schwer Einhalt geboten werden konnte. In der SBZ versuchte die ZVAS unter anderem mit arbeitsrechtlichen Bestimmungen das gesamtwirtschaftliche Problem in den Griff zu bekommen. In einem Rundschreiben an die Landesarbeitsämter und Arbeitsämter wies die Berliner Zentralverwaltung am 8. November 1945 darauf hin, daß grundsätzlich niemand daran gehindert werden solle, „sich eine selbständige Existenz zu sichern" 84 . Bei vielen seien allerdings andere Gründe ausschlaggebend: Dazu zählte die ZVAS das Bestreben, dem Arbeitseinsatz auszuweichen, zusätzliche Nahrungsmittel zu beziehen und „Freizügigkeit zu erlangen, um sich auf dem schwarzen Markt betätigen zu können.“ Daher sollte die Gewerbeerlaubnis nur nach sorgfältiger Prüfung und nach Einschaltung des zuständigen Arbeitsamtes erteilt werden. Als Scheinarbeitsverhältnis definierte die ZVAS-Abteilung II am 26. Februar 1946 jede Vereinbarung, „die ein Arbeitsverhältnis vortäuscht, während tatsächlich zwischen den Vertragspartnern weder Arbeit gefordert noch ge-

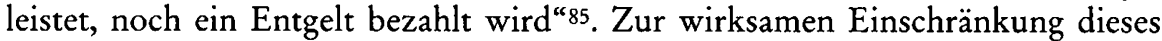
Phänomens sprach sich die Berliner Zentralverwaltung dafür aus, regelmäßige und sorgfältige Prüfungen der Arbeitsbücher sowie Betriebskontrollen durchzuführen ${ }^{86}$.

Die ZVAS hatte zwar in den Richtlinien über die Registrier- und Meldepflicht vom 25. Oktober 1945 unmißverständlich klargestellt, daß Scheinarbeitsverhältnisse keine Beschäftigungsverhältnisse darstellten. Den Ämtern für Arbeit und Sozialfürsorge reichte allerdings die bestehende Rechtslage nicht aus; sie verlangten die Ausarbeitung einer Verordnung mit weitreichenden Vollmachten, vor allem mit der Einführung von Strafbestimmungen ${ }^{87}$. Nach Beratung mit dem zuständigen Abteilungsleiter Kreil verfügte Präsident Brack die Vorbereitung eines Gesetzentwurfes, der den Landes- und Provinzialregierungen zur Beschlußfassung durch die Landtage vorzulegen war ${ }^{88}$. Die DVAS-Abteilung I b legte am 8. Februar 1947 einen Gesetzentwurf zur Bekämpfung von Scheinarbeitsverhältnissen vor ${ }^{89}$ : Danach sollten die Sanktionen sowohl die Auftraggeber als auch die Auftragnehmer treffen und konnten im Höchstfall eine Gefängnisstrafe von sechs Monaten bzw. eine Geldstrafe von 10000,- RM erreichen. Eine inhaltliche Kontroverse bahnte sich erneut mit der Juristischen Abteilung an, die nur eine Verordnung erlassen wollte und außerdem für eine enge Auslegung des Begriffes "Scheinarbeitsverhältnis“ eintrat ${ }^{90}$. Als problematisch erwies sich ferner der Versuch, einheitlich über die Landtage ein entsprechendes Gesetz verabschieden zu lassen, da somit unmittelbar in die noch bestehende Länderhoheit eingegriffen wurde. Dazu schlug die DVAS ein eng abgestimmtes Vorgehen zwischen den

84 BAB, DQ 2/2064, ZVAS-Abt. II (Kreil) am 8. 11. 1945 an das LAA Mecklenburg-Vorpommern.

s5 BAB, DQ 2/1738, Notiz vom 26. 2. 1946, S. 2.

86 Ebenda, S. 3.

87 BAB, DQ 2/1746, Aktennotiz der DVAS-Abt. I b vom 4. 2. 1947, S. 2.

88 Ebenda, S. 3.

89 BAB, DQ 2/2194.

$90 \mathrm{BAB}, \mathrm{DQ}$ 2/2194, Aktennotiz der DVAS-Abt. I b vom 16. 2. 1947. 
Landesämtern und der Zentralverwaltung in Berlin vor, das sich auf alle sozialpolitischen Gesetzesvorhaben erstrecken sollte ${ }^{91}$. DVAS-Präsident Brack übersandte den zuständigen Ministern der Landes- bzw. Provinzialregierungen am 3. März den Gesetzentwurf mit der Bitte, diesen durch den Landtag verabschieden zu lassen ${ }^{92}$. Die weitere Entwicklung zeigte, daß eine einheitliche Gesetzesregelung in den Ländern nicht so ohne weiteres zustande kommen würde. So hielt etwa das mecklenburgische Ministerium für Sozialwesen ein „besonderes Strafgesetz" nicht für erforderlich"3. Als „wirksame Erziehungsmaßnahme" sei vielmehr auf die Zwangseinweisung zurückzugreifen, „wenn notwendig in eine Arbeitskolonne mit niedriger Arbeit oder ungünstigen Umständen." Dagegen nahm der sächsische Landtag den Gesetzentwurf am 6. Juni an ${ }^{94}$. In Thüringen zog sich das Verfahren noch etwas länger hin, da die Landesregierung den zugesandten $\mathrm{Ge}-$ setzentwurf überarbeitete und teilweise verschärfte: So wurde bereits der Versuch, ein Scheinarbeitsverhältnis einzugehen, unter Strafe gestellt. Darüber hinaus unterschied der thüringische Entwurf bei den Strafbestimmungen zwischen schwereren und leichteren Tatbeständen 95 .

Die Deutsche Justizverwaltung unterrichtete die DVAS am 9. September 1947 über das geplante Gesetzesvorhaben in Thüringen und bat um eine Stellungnahme ${ }^{96}$. Offensichtlich war die DVAS von der thüringischen Landesregierung nicht informiert worden; anders läßt sich das Antwortschreiben ihres Präsidenten Brack nicht erklären ${ }^{97}$. Dieser kritisierte den thüringischen Entwurf allerdings nicht wegen seiner inhaltlichen Verschärfung. Sein Einwand ging in eine andere Richtung: Die Strafbestimmungen müßten auch auf die Unternehmerseite bezogen werden. Das habe die thüringische Landesregierung bei ihrer Überarbeitung fallengelassen. Ende September war der Streit zwischen der DVAS und der thüringischen Landesverwaltung, die auf ihrem Entwurf beharrte, noch nicht beigelegt. In der Zwischenzeit war in Mecklenburg in Verbindung mit der dortigen Justizverwaltung eine „Polizeiverordnung“ erlassen worden, um auf diesem Wege die Zustimmung des Landtages zu umgehen ${ }^{98}$. Die brandenburgische Landesverwaltung hatte zu diesem Zeitpunkt bereits eine Gesetzesvorlage in den Landtag eingebracht, während in Sachsen-Anhalt noch gar keine Initiative entfaltet worden war.

Die DVAS registrierte im Oktober 1947 eine nicht unerhebliche Diskrepanz zwischen der Zahl der registrierten Erwerbstätigen und der der registrierten Versicherten; das betraf sowohl die unselbständig Beschäftigten als auch die selbständig Beschäftigten 99 . So waren in der SBZ bei den Arbeitsämtern am 31. Juli 1947 insgesamt 5891055 unselbständig Beschäftigte gemeldet, bei den Sozialversiche-

91 Ebenda, Aktennotiz der DVAS-Abt. I b vom 24. 2. 1947.

92 BAB, DQ 2/2194.

93 Ebenda, Ministerium für Sozialwesen in Schwerin am 8. 4. 1947 an die DVAS.

94 Ebenda, Landesarbeitsamt Sachsen am 21.6. 1947 an die DVAS.

95 Ebenda, Ministerium für Wirtschaft, Arbeit und Verkehr in Weimar am 9.7.1947 an die DVAS.

$96 \mathrm{BAB}, \mathrm{DQ} 2 / 1820$.

$97 \mathrm{BAB}, \mathrm{DQ} 2 / 1820$, Präsident Brack am 23. 9. 1947 an den Chef der Deutschen Justizverwaltung.

$98 \mathrm{BAB}, \mathrm{DQ} 2 / 1537$, Niederschrift über den Verlauf der Arbeitstagung in Neuruppin (25.-27.9. 1947), S. 15.

99 Zu den folgenden Zahlen: BAB, DQ 2/1711, Vermerk der DVAS-Abt. I a (Donau) vom 1. 10. 1947 an Präsident Brack. 
rungsanstalten allerdings nur 5131570 Versicherte. Darunter befanden sich 175679 Männer und immerhin 583806 Frauen. Vergleichsweise größer waren die Unterschiede bei den Selbständigen, wobei in dieser Gruppe auch die mithelfenden Familienangehörigen erfaßt wurden: Hier zählte die Arbeitsverwaltung 1783856 Personen, denen nur 1099408 Versicherte gegenüberstanden. Wo lagen die Ursachen dafür? Gerade in der Landwirtschaft waren zahlreiche Beschäftigte nicht versicherungspflichtig, weil sie aufgrund des Verdienstes oder der Dauer des Arbeitsverhältnisses nicht unter die Bestimmungen der Sozialpflichtversicherung fielen. Darüber hinaus wurden versicherungspflichtige Personen vom Arbeitgeber oftmals nicht versichert; hinzu kamen die mithelfenden Familienangehörigen, die von der DVAS wie auch die beiden erstgenannten Gruppen nicht quantifiziert werden konnten. Zur geringeren Zahl der bei der Sozialversicherung gemeldeten Personen trug außerdem bei, daß Beschäftigte bei den sowjetischen Dienststellen zumeist nicht versichert waren. Als letztes sind die Frauen zu nennen, die mit ihrem Verdienst aufgrund einer geringfügigen Beschäftigung unter dem Mindestsatz der Sozialversicherung lagen. DVAS-Präsident Brack leitete diesen Bericht an die SMAD-Abteilung Arbeitskraft weiter und machte deutlich, daß die Arbeitsämter auch in Zusammenarbeit mit den Ernährungsämtern nicht in der Lage waren, eine Klärung herbeiführen ${ }^{100}$. Die Kommunalverwaltung sei „in allzu starkem Ausmaße mit der Bevölkerung verbunden“, so daß der Aufbau eines eigenen „Kontrollapparates“ erforderlich sei, „der völlig unabhängig und durch keinerlei Bindungen in seiner Arbeit gehemmt" werde. Die DVAS hatte sich in dieser Frage mit der Deutschen Verwaltung für Handel und Versorgung bereits abgesprochen und schlug der SMAD vor, zehn Inspektoren zusätzlich einzustellen, die den Arbeitsschutzinspektoren in der Besoldung gleichgestellt werden sollten. Die SMADFinanzabteilung lehnte diesen Vorstoß $\mathrm{ab}^{101}$.

Anfang 1948 äußerte der Leiter der DVAS-Abteilung I b Kreil in einem hausinternen Vermerk Zweifel an den Erfolgsaussichten des eingeschlagenen Weges, den er sicherlich zutreffend als zu „zeitraubend“ einschätzte ${ }^{102}$. Immerhin war seit der Zustellung des Gesetzentwurfes an die Landesverwaltungen fast ein Jahr verstrichen, ohne daß eine einheitliche Regelung gefunden werden konnte. Auf diese Weise sei ein Gesetz für die SBZ „nie zu einer einheitlichen Durchführung zu bringen“, so die realistische Einschätzung Kreils. Etwas frustriert stellte er abschließend noch die Frage: „Wenn schon bei einem so einfachen Gesetz, das nur zwei fachliche Paragraphen umfaßt, die Meinungen in der Beurteilung soweit voneinander abweichen, wie soll es dann gelingen, ein Gesetz von einigen dutzend Paragraphen ordnungsgemäß unter Dach und Fach zu bringen. “ Dieser Meinung schloß sich die Juristische Abteilung an, die an allen vorliegenden Entwürfen der einzelnen Länder inhaltliche Kritik übte, und sogar noch einen Schritt weiter ging, indem sie sich dafür aussprach, „auf das Gesetz überhaupt zu verzichten“103.

Letztlich verzichtete die DVAS darauf, ein eigenes Gesetz gegen Scheinarbeitsverhältnisse zu verabschieden, sei es als Gesetz durch die Landtage oder als Ver-

100 BAB, DQ 2/1711, Brack am 2. 10. 1947 an Morenow.

101 Ebenda, Aktenvermerk der DVAS-Abt. I b vom 4. 11. 1947.

$102 \mathrm{BAB}, \mathrm{DQ} 2 / 2194$, DVAS-Abt. I b am 30. 1. 1948 an Juristische Abt.

${ }^{103} \mathrm{BAB}, \mathrm{DQ} 2 / 111, \mathrm{Bl} .86$ f., hier Bl. 87, Juristische Abt. am 3. 2. 1948 an Abt. I b. 
ordnung durch die Zentralverwaltung. Dabei spielte wohl auch die Überlegung eine Rolle, daß sich bei der Umsetzung einer solchen rechtlichen Bestimmung erhebliche Schwierigkeiten für die Kommunalverwaltung ergeben würden. So stellte etwa die Juristische Abteilung der DVAS die Frage, wie die Verwaltungsstellen, welche für die Ausgabe der Lebensmittelkarten verantwortlich zeichneten, das Vorliegen von Scheinarbeitsverhältnissen überprüfen sollten ${ }^{104}$. Die SMAD-Abteilung Arbeitskraft drängte zwar des öfteren auf eine umfassende Kontrolltätigkeit von seiten der Landesverwaltungen ${ }^{105}$, intervenierte aber nicht bei der Diskussion über die Ausarbeitung eines Gesetzes durch die Landtage. Für die sowjetische Besatzungsmacht war die reibungslose Tätigkeit der Arbeitsämter und die Eindämmung der Scheinarbeitsverhältnisse von entscheidender Bedeutung, die Wahl der Mittel blieb - so hat es den Anschein - der deutschen Arbeitsverwaltung überlassen. Die zahlenmäßige Erfassung der Personengruppe, die sich der Registrierung entzog, bereitete den Arbeitsämtern immer noch große Probleme. Ende 1948 lagen nur für Brandenburg erste Ergebnisse vor. Dort ging das zuständige Landesarbeitsamt von über 20000 Personen aus, die in einem Scheinarbeitsverhältnis standen ${ }^{106}$.

\section{Verordnung betr. eine Erbebung über Fluktuation, Arbeitsdisziplin und Leistung}

Die Binnenwanderung innerhalb der SBZ, aber auch die Fluktuation erwiesen sich für die Arbeitsverwaltung in der unmittelbaren Nachkriegszeit als ein zentrales Problem, dem Einhalt geboten werden sollte. Die zentrale Registrierung des Arbeitskräftepotentials schien dadurch gefährdet zu sein. Aus diesem Grunde brachte die DVAS am 1. November 1947 eine Verordnung heraus, die alle Unternehmer mit mehr als 100 Beschäftigten verpflichtete, einmal monatlich eine Arbeitsstatistik beim zuständigen Arbeitsamt einzureichen ${ }^{107}$. Diese Berichte sollten genaue Angaben über die Veränderung des Beschäftigtenstandes, der ausgefallenen Arbeitszeit sowie eine Leistungsübersicht enthalten. Unmittelbar zuvor hatte die SMAD den Befehl Nr. 234 über „Maßnahmen zur Steigerung der Arbeitsproduktivität und zur weiteren Verbesserung der materiellen Lage der Arbeiter und Angestellten in der Industrie und im Verkehrswesen " veröffentlicht ${ }^{108}$, der den inhaltlichen Hintergrund für die neue Verordnung bildete. Durch ein kombiniertes System von leistungsbezogenen Löhnen ${ }^{109}$, betrieblicher Sozialpolitik und Kontrollen zur Senkung des Krankenstandes ${ }^{110}$ sollte insgesamt die Produktivität in den Betrieben gesteigert werden. Dazu schien zunächst einmal eine detaillierte statistische Erhebung erforderlich zu sein.

104 BAB, DQ 2/70, Aktenvermerk der Juristischen Abt. vom 15. 3. 1948.

$105 \mathrm{BAB}, \mathrm{DQ} 2 / 1950$, Aktennotiz der Abt. I b über die Besprechung bei der SMAD in Karlshorst am 3. 11. 1948, S. 2 .

106 Ebenda.

107 Arbeit und Sozialfürsorge 2 (1947), S. $471 \mathrm{f}$.

108 Ebenda, S. $452 \mathrm{f}$.

109 Vgl. Hübner, Konsens, Konflikt und Kompromiß, S. 21-27.

110 Vgl. Hoffmann, Sozialpolitische Neuordnung, S. 81-85. 
Bereits am 5. November 1947 informierte die DVAS sämtliche Arbeitsämter in einem Rundschreiben über die Verordnung und erteilte die Anweisung, die Durchführung zu überprüfen ${ }^{111}$. Die DVAS teilte in dem Zusammenhang mit, daß die Gründe für die Fluktuation "größtenteils“ bekannt seien, „doch kommt es jetzt darauf an, das tatsächliche Ausmaß in den verschiedenen Wirtschaftszweigen festzustellen". Dazu sollten die Arbeitsämter vorgefertigte Berichtsmuster an alle Betriebe innerhalb ihres Zuständigkeitsbereiches versenden, die mehr als $100 \mathrm{Be}-$ schäftigte hatten.

Bereits im Sommer 1948 wurde diese Verordnung von der DWK wieder aufgehoben, da die in der Zwischenzeit durch den SMAD-Befehl Nr. 282 vom 26. Dezember 1947 geänderte Betriebsberichtserstattung eine weitere zusätzliche statistische Erhebung überflüssig gemacht hatte ${ }^{12}$. Damit war die Verordnung vom 1. November 1947 de facto außer Kraft gesetzt worden, zumal die örtlichen Industrieämter, die Industrie- und Handelskammern sowie die Statistischen Kreis- und Landesämter die Zusammenstellung des Zahlenmaterials übernehmen mußten. Für die Arbeitsverwaltung bedeutete dies auch eine Arbeitsentlastung. Das Sekretariat der DWK verabschiedete auf der Sitzung am 11. August 1948 die von der HVAS ausgearbeitete Verordnung 113 .

Eine ganz besondere Form der Fluktuation stellte die Abwanderung von Arbeitskräften in die westlichen Besatzungszonen dar, die von der Arbeitsverwaltung, aber auch von SED und SMAD aufmerksam beobachtet wurde. Die Abwanderung in den Westen kann als Vorläufer der erst zu Beginn der fünfziger Jahre abqualifizierten und verfolgten "Republikflucht " gewertet werden, und dennoch kommt ihr in der Zeit der unmittelbaren Besatzungsherrschaft bis zur Gründung der DDR 1949 eine eigene Spezifik zu. Diese beruhte auf den sozialen und wirtschaftlichen Umwälzungen der Zusammenbruchsgesellschaft. Insofern muß die Abwanderung in den Westen Deutschlands in den ersten Nachkriegsjahren als Bestandteil der allgemeinen Bevölkerungswanderung gesehen werden; sie war sehr stark von wirtschaftlichen Faktoren geprägt, erhofften sich doch die Abwandernden bessere Arbeitsbedingungen als in der SBZ. Frühzeitig brachte die SMAD diese berufliche Migration mit gezielter Abwerbung von seiten westdeutscher Unternehmer, ja sogar von seiten westlicher Arbeitsämter in Verbindung. So erklärte der stellvertretende Leiter der Abteilung Arbeitskräfte Morenow, daß sich das Landesarbeitsamt Bremen „anmaßt, Arbeitskräfte für Mangelberufe in den einzelnen Ländern und Provinzen der Sowjetzone zu werben"114. Morenow wollte diese Angelegenheit beim Alliierten Kontrollrat zur Sprache bringen. Die vier Siegermächte konnten sich jedoch allem Anschein nach nicht auf ein einheitliches Vorgehen in dieser Frage einigen, so daß es der SMAD und den deutschen Arbeitsverwaltungen überlassen blieb, Gegenmaßnahmen zu ergreifen. Möglichkeiten zur wirksamen Einschränkung dieser Wanderungsbewegung bestanden ohnehin kaum. So stellte die DVAS im Frühjahr 1947 fest: „Wenn einer der Alli-

111 BAB, DQ 2/1754, Rundschreiben Nr. 177 der DVAS vom 5. 11. 1947.

$112 \mathrm{BAB}, \mathrm{DC}$ 15/II/1-6, Bl. 75, Begründung der Verordnung über die Einstellung der Erhebung über Fluktuation, Arbeitsdisziplin und Leistung.

113 BAB, DC 15/344, Bl. 2.

114 BAB, DQ 2/67, Bl. 9, Aktenvermerk über Besprechung mit der SMAD (Morenow) am 27. 2. 1946. 
ierten Staaten durch einen Beauftragten Fachkräfte anwirbt, so können wir dies zur Zeit nicht [ver]hindern." 115 Gleichwohl betonte die DVAS, daß „es nicht wünschenswert ist, die Auswanderung von qualifizierten Fachkräften durch irgendwelche tätige Unterstützung oder Auskunftserteilung zu fördern." Dieses Problem erstreckte sich nicht nur auf die Wanderung nach Westdeutschland, sondern auch auf die Abwanderung ins Ausland. Der Kontrollratsbefehl Nr. 3 hatte den Arbeitsämtern eine Monopolstellung bei der Arbeitsvermittlung und -lenkung zugeschrieben; damit war eine Werbung außerhalb der Arbeitsämter faktisch nicht zugelassen. Nach Einschätzung der Berliner Zentralverwaltung untersagte außerdem die Proklamation Nr. 2 des Alliierten Kontrollrates allen Deutschen, „ohne Erlaubnis oder Befehl der alliierten Vertreter deutsches Gebiet zu verlassen" 116 . Diese Bestimmung war allerdings recht vage gehalten, und die Siegermächte hatten auch im Frühjahr 1947 kein ausdrückliches Verbot der Abwerbung durch ausländische Staaten erlassen.

Eine Quantifizierung dieser Wanderungsbewegung ist für die ersten Nachkriegsjahre nicht möglich, da die Arbeitsämter dieses Phänomen nicht systematisch erfassen konnten. Vielmehr blieb es bei Einzelmeldungen, die zumindest die Schlußfolgerung zulassen, daß die betroffenen Zentral- und Landesverwaltungen in der SBZ die Abwanderung durchaus ernst nahmen. So berichtete ein Mitarbeiter der SED-Bezirksleitung Berlin im Herbst 1947, daß sich in Berlin-Heiligensee eine französische "Werbungsstelle" für ostdeutsche Facharbeiter befinde, die täglich 120 Bewerber beraten und weiter vermitteln würde ${ }^{117}$. Auf diese Weise seien bisher 565 Facharbeiter in das Saargebiet und 1120 weitere Facharbeiter in die französische Besatzungszone abgewandert; 90 Prozent dieser Gruppe stammten offensichtlich aus der SBZ bzw. aus dem russischen Sektor Berlins. Neben Berlin waren vor allem einige Bezirke in Thüringen betroffen: So meldete das dortige Landesarbeitsamt, daß im November 1947 ein Sonderzug aus Jugoslawien in Ilmenau eingetroffen sei, der mit 100 angeworbenen Arbeitskräften der Glasindustrie und deren Familienangehörigen wieder abgefahren sei118. Die thüringische SMA hielt sich auffallend zurück und weigerte sich im letztgenannten Fall sogar, ein Werbungsverbot zu verhängen ${ }^{119}$. Aufschlußreich war die Tatsache, daß die SMAD in Karlshorst keine Informationen über die Vorgänge in Thüringen besaß und erst durch die Berliner Zentralverwaltung darüber in Kenntnis gesetzt worden war. Nach Angaben der DVAS betrieben Frankreich, das für seine äußerst restriktive Einwanderungspolitik in die eigene Besatzungszone bekannt war, und Schweden eine gezielte Pressepropaganda, um Facharbeiter zu gewinnen.

Die DVAS konnte die angebliche Anwerbung von Arbeitskräften durch ausländische Firmen nur registrieren und alle Arbeitsämter anweisen, „alle Beobachtungen" umgehend mitzuteilen. Anschließend sollten die gesammelten Berichte der

$115 \mathrm{BAB}, \mathrm{DQ} 2 / 2058$, Abteilungsleiter Kreil am 17. 3. 1947 an das thüringische Ministerium für Wirtschaftsplanung.

116 BAB, DQ 2/2009, Abschnittsleiter Kreil am 20. 5. 1947 an Josef F.

117 SAPMO, NY 4090/561, Bl. 29, Aktennotiz Stephanowitz für Otto Grotewohl vom 22. 9. 1947.

118 BAB, DQ 2/2040, Bl. 108, Niederschrift über Besprechung mit der SMAD am 4. 12. 1947.

119 Ebenda, Bl. 109. 
SMAD unterbreitet werden ${ }^{120}$. Auch der FDGB-Bundesvorstand wies aufgrund von Berichten seines thüringischen Landesverbandes darauf hin, daß in Thüringen "systematisch Werber aus den westlichen Zonen und von ausländischen Firmen auftreten, die Fachkräfte anzuwerben versuchen "121. Die HVAS wurde gebeten, konkrete Maßnahmen zu treffen, daß „uns das wertvolle Kapital der Fachkräfte, besonders in den Spezialindustrien, erhalten bleibt." Nach Ansicht des Gewerkschaftsbundes spielte die Ernährungsfrage ein entscheidendes Motiv bei der Abwanderung aus der SBZ. Der SMAD-Befehl Nr. 234 vom Oktober 1947 hatte bereits eine Verbesserung der Lebensmittelversorgung für ausgewählte Berufsgruppen anvisiert. Nach den Vorstellungen des FDGB sollten diese Bestimmungen auch auf andere Berufsgruppen ausgedehnt werden, um die Migration in die Westzonen zu unterbinden. Die HVAS hielt es letztlich für „unzweckmäßig“, eine eigene Rundverfügung in dieser Angelegenheit zu erlassen und verwies zur Begründung auf den Kontrollratsbefehl Nr. 3, der die „illegale“ Werbung bzw. Vermittlung unter Strafe gestellt hatte ${ }^{122}$. Vor allem die SMAD zeigte wenig Interesse daran, neue gesetzliche Bestimmungen auszuarbeiten und zu veröffentlichen. Die Abteilung Arbeitskraft in Karlshorst sprach sich zwar dafür aus, „gegen derartige illegale Werbung vor[zu]gehen "123. Dies sollte aber ausschließlich über entsprechende Propagandaaktivitäten und Pressemitteilungen erfolgen, die von den Arbeitsämtern zu veröffentlichen waren. Die SMAD schlug in dem Zusammenhang vor, „diejenigen Personen sprechen zu lassen, die aus Frankreich zurückgekehrt sind und über die wahren Zustände dort Auskunft geben können."

Die gezielte Abwerbung von Arbeitskräften wurde aber auch von Firmen und Unternehmen innerhalb der SBZ praktiziert, die eigens dafür sogenannte Werber einsetzten. Dagegen versuchte die DVAS frühzeitig vorzugehen. Ihre Handlungsspielräume waren eng gesteckt, sobald es sich um SAG-Betriebe handelte oder um Betriebe, die für die sowjetische Besatzungsmacht produzierten. Daran läßt sich unter anderem die stellenweise mangelhafte Absprache zwischen deutschen und sowjetischen Dienststellen ablesen. So kritisierte etwa das Arbeitsamt Halberstadt, daß ein Arbeitseinsatzleiter vom Eisen- und Hüttenwerk Thale/Harz versucht habe, ausgebildete Schweißer anzuwerben ${ }^{124}$. Außerdem zeigte sich das Arbeitsamt, das dieses Ersuchen abgelehnt hatte, darüber verärgert, daß dies unter der Androhung geschah, die Kreiskommandantur einzuschalten, um von dort die Abstellung der Arbeitskräfte zu erwirken. Das Ministerium für Arbeit und Sozialpolitik von Sachsen-Anhalt stellte ohne Umschweife fest, daß das Eisen- und Hüttenwerk Thale „in keiner Weise berechtigt [war], derartige Abwerbungen vorzunehmen " 125 . Es bestehe zwar ein großer Arbeitskräftebedarf; dies dürfe jedoch nicht dazu führen, „daß Handlungen vorgenommen werden, die jeder recht-

$120 \mathrm{BAB}, \mathrm{DQ} 2 / 1537$, Niederschrift über den Verlauf der Arbeitstagung der DVAS (Abt. I b) in Leipzig am 6./7. 1. 1948, S. $26 \mathrm{f}$.

121 BAB, DQ 2/2009, FDGB-Bundesvorstand (HA 2: Wirtschaftspolitik) am 1.6. 1948 an die HVAS.

122 Ebenda, HVAS am 29. 6. 1948 an FDGB-Bundesvorstand (HA 2).

$123 \mathrm{BAB}, \mathrm{DQ} 2 / 2040$, Bl. 143, Aktennotiz über Besprechung bei der SMAD am 1. 7. 1948.

124 BAB, DQ 2/2009, Amt für Arbeit und Sozialfürsorge Halberstadt am 13. 9. 1947 an das Ministerium für Arbeit und Sozialpolitik der Landesregierung Sachsen-Anhalt.

125 Ebenda, Ministerium für Arbeit und Sozialpolitik der Landesregierung Sachsen-Anhalt am 18.9. 1947 an das Eisen- und Hüttenwerk Thale. 
lichen Grundlage entbehren“. Die angegriffene Werksleitung von Thale ging nunmehr in die Offensive und erklärte, daß „es im Rahmen der allgemeinen Verpflichtung unserer Wirtschaft mit dem Ziel, die Arbeitskräfte dahin zu bringen, wo sie am notwendigsten gebraucht werden, für uns durchaus nicht , selbstverständlich ist, daß ein derartiges Ersuchen [Arbeiter anzuwerben] verweigert worden ist" 126. Mit leicht süffisantem Unterton rechtfertigte die Direktion von Thale die Androhung, gegebenenfalls die sowjetische Kreiskommandantur einzuschalten, denn schließlich sei die Anweisung direkt von der SMAD in Karlshorst gekommen. Damit mußte die Landesregierung von Sachsen-Anhalt, die über diese Rückendeckung nicht unterrichtet worden war, klein beigeben.

\section{„Arbeitsmarkt"127 und Arbeitslosigkeit}

Neben der Registrierung der erwerbsfähigen Bevölkerung bestand eine weitere Hauptaufgabe der Arbeitsverwaltung darin, regelmäßig über Disproportionen und Anpassungsschwierigkeiten auf dem Arbeitsmarkt zu berichten. Somit rückte die Arbeitslosigkeit in das Zentrum des Interesses der DVAS/HVAS und nahm einen herausragenden Stellenwert in der Verwaltungstätigkeit bis zur DDRGründung 1949 ein. Im einzelnen sollten monatlich und quartalsweise ausführliche Berichte erstellt werden, die unter anderem der sowjetischen Besatzungsmacht zugesandt wurden. Die SMAD-Abteilung Arbeitskraft hatte ein großes Interesse an diesen Informationen und verlangte beispielsweise am 16. Oktober 1945 „Unterlagen über den Stand der Arbeitslosigkeit in den einzelnen Provinzen und Ländern, nach Berufen aufgeteilt"128. Wenige Tage später betonte die SMAD, daß die Arbeitslosigkeit nicht nur "genau“ festzustellen, sondern in Zusammenarbeit mit den zuständigen "Wirtschaftsstellen" zu beseitigen sei. Begründet wurde diese Vorgabe mit dem Hinweis, „daß es Arbeitslose nicht geben soll“129. Ende November empfahl die Abteilung Arbeitskraft, nur solche Personen als Arbeitslose zu registrieren, „die unmittelbar zur Arbeitsvermittlung zur Verfügung stehen" ${ }^{130}$. Dagegen sollten die Arbeitspflichtigen, welche „aus familiären Gründen oder aus Krankheitsgründen oder weil sie sich in der Umschulung befinden“, nicht erfaßt werden. Dieser Vorschlag war allerdings überflüssig, da die deutsche Arbeitsverwaltung eine solche statistische Erhebung überhaupt nicht vorgenommen hatte.

Im folgenden Abschnitt wird nicht der Versuch unternommen, eine umfassende und in sich geschlossene Darstellung zur Entwicklung des ostdeutschen Arbeitsmarktes zwischen Kriegsende und DDR-Gründung zu geben ${ }^{131}$. Vielmehr

126 Ebenda, Eisen- und Hüttenwerke Thale am 30. 9. 1947 an das Ministerium für Arbeit und Sozialpolitik der Landesregierung Sachsen-Anhalt.

${ }_{127}$ Auf die Schwierigkeiten bei der Verwendung dieses Begriffes für die SBZ/DDR ist bereits in der Einleitung hingewiesen worden.

128 $\mathrm{BAB}, \mathrm{DQ} 2 / 1, \mathrm{Bl} .6$, Aktenvermerk über Besprechung mit Remissow in Karlshorst am 16. 10. 1945.

${ }^{129}$ Ebenda, Bl. 7, Aktenvermerk über Besprechung mit Remissow und Morenow in Karlshorst am 24. 10. 1945.

130 Ebenda, Bl. 15, Aktenvermerk über Besprechung mit Remissow in Karlshorst am 28.11. 1945.

131 Vgl. dazu etwa Zank, Wirtschaft und Arbeit, S. 30-57, 170-181. 
sollen einige zentrale Problemfelder kurz skizziert werden, die aus Sicht der Arbeitsverwaltung die Lenkung der Arbeitskräfte beeinflußt und vor allem erschwert haben. Dadurch lassen sich wiederum Rückschlüsse auf die Handlungsspielräume der Arbeitsämter in dieser Periode ziehen. Ein enorm wichtiger Faktor wird an dieser Stelle zunächst nicht weiter untersucht, sondern erst im folgenden Kapitel vorgestellt: der Einfluß der sowjetischen Besatzungsmacht und deren Arbeitskräfteanforderungen.

Der Zweite Weltkrieg hatte in den drei westlichen wie auch in der sowjetischen Besatzungszone zu einer erheblichen Veränderung der Bevölkerungsstruktur geführt. Die Verluste unter der Zivilbevölkerung waren zwar durch den millionenfachen Zustrom von Flüchtlingen und Vertriebenen mehr als ausgeglichen worden, so daß sich im Oktober 1946 bei der Volkszählung in der SBZ etwa 1,1 Millionen Menschen mehr aufhielten als noch 1939. Dagegen war jedoch die Gesamtzahl der arbeitsfähigen Menschen im gleichen Zeitraum um rund 400000 gesunken. Erst Anfang der fünfziger Jahre schlug die Arbeitskräfteknappheit in der SBZ in einen Arbeitskräfteüberschuß um: Vor allem die „Umsiedler“, aber auch die aus der Kriegsgefangenschaft heimkehrenden Männer trugen - wie bereits anfangs erwähnt - zu diesem Prozeß bei. Nach Angaben von Wolfgang Zank war das Arbeitskräftepotential um 1950 sowohl quantitativ als auch qualitativ, was das Angebot an Facharbeitern betraf, ein Überschußbereich ${ }^{132}$. Ab 1950 setzte dann bis zum Mauerbau am 13. August 1961 ein Rückgang der Bevölkerungszahl ein, der in erster Linie auf die massive Fluchtbewegung von mindestens 2,7 Millionen Menschen in den Westen Deutschlands zurückzuführen ist. Der DDR gingen dadurch in hohem Maße junge Menschen und qualifizierte Fachkräfte verloren.

In der SBZ registrierte die Arbeitsverwaltung im IV. Quartal 1945 zwischen 536000 und 590000 Arbeitslose ${ }^{133} ; 1946$ sank diese Zahl im langfristigen Trend bis auf 182000 im September. Diese Entwicklung setzte sich 1947 fort und erreichte einen Tiefstand von 102000 (August 1947); 1948 erfolgte eine leichte Trendwende, bei der die Arbeitslosenzahlen wieder etwas anstiegen. Insgesamt gesehen konnten in den beiden Jahren 1947/48 die Erwerbslosenzahlen auf einem Monatsniveau zwischen 102000 und 231000 gehalten werden. Ende 1948 stiegen die Erwerbslosenzahlen wieder kontinuierlich an ${ }^{134}$ und erreichten im März 1949 eine

132 Zank, Wirtschaft und Arbeit, S. 57.

${ }_{133} \mathrm{Vgl}$. zu den Zahlen auch die folgende Tabelle 1.

${ }^{134}$ Durch die Währungsumstellung und die damit einhergehende Verknappung der Geldmenge waren vor allem kleinere und mittlere Betriebe in Mitleidenschaft gezogen worden, da deren vergleichsweise dünne Kapitaldecke nunmehr vollends zusammenzubrechen drohte. Von dieser Entwicklung waren auch zahlreiche Flüchtlingsbetriebe betroffen. Langfristig betrachtet ging damit auch ein Rückgang der Selbständigen in der SBZ/DDR einher. Eine weitere Folge war außerdem das Zurückgehen der bei den Arbeitsämtern registrierten offenen Stellen, da zahlreiche Betriebe keine weiteren Arbeitskräfte mehr nachfragten. Damit wurde ein Großteil der Arbeitsuchenden, die erst nach der Währungsumstellung auf den Arbeitsmarkt strömten, als Arbeitslose registriert. Vgl. BAB, DQ 2/1888, Niederschrift der HVAS vom 29. 3. 1949 über das Ergebnis der Untersuchungskommission im Bereich des Arbeitsamtes Magdeburg. Vgl. zur Währungsreform in der SBZ: Zschaler, Die vergessene Währungsreform. Die Haltung der Sowjetunion in dieser Frage beleuchtet Laufer, Die UdSSR und die deutsche Währungsfrage. Ein Ansteigen der Arbeitslosenzahlen zeichnete sich im übrigen auch in den drei Westzonen ab. Vgl. dazu: Erker, Ernährungskrise und Nachkriegsgesellschaft, S. 263-267; Woller, Gesellschaft und Politik, S. 301 f. Buchheim hat 
Rekordmarke von 390000, die zuletzt im Februar 1946 höher gelegen hatte (435000). Als wichtigste Ursachen für die ansteigende Arbeitslosigkeit lassen sich folgende Faktoren ausmachen: die zu diesem Zeitpunkt sich herausbildende strukturelle Arbeitslosigkeit, die statistische Verschiebung von Unterstützungsempfängern zu den meldepflichtigen Arbeitslosen ${ }^{135}$ sowie die anhaltende Entlassung von Kriegsgefangenen aus den sowjetischen Lagern, die auf den deutschen Arbeitsmarkt zurückströmten. Darüber hinaus wirkten sich der Rohstoff- und Materialmangel hemmend auf die Produktion und damit indirekt auf die Beschäftigungslage in den Betrieben aus ${ }^{136}$. Erst 1951 erreichte die DDR Arbeitslosenzahlen ${ }^{137}$, die unter 200000 lagen, und es sollte noch ein weiteres Jahr dauern, bis erstmals Werte unter 100000 registriert wurden (Mai 1952).

Grafik 1: Entwicklung der Arbeitslosigkeit in der SBZ (obne Berlin) 1945-1949 (in Tausend)

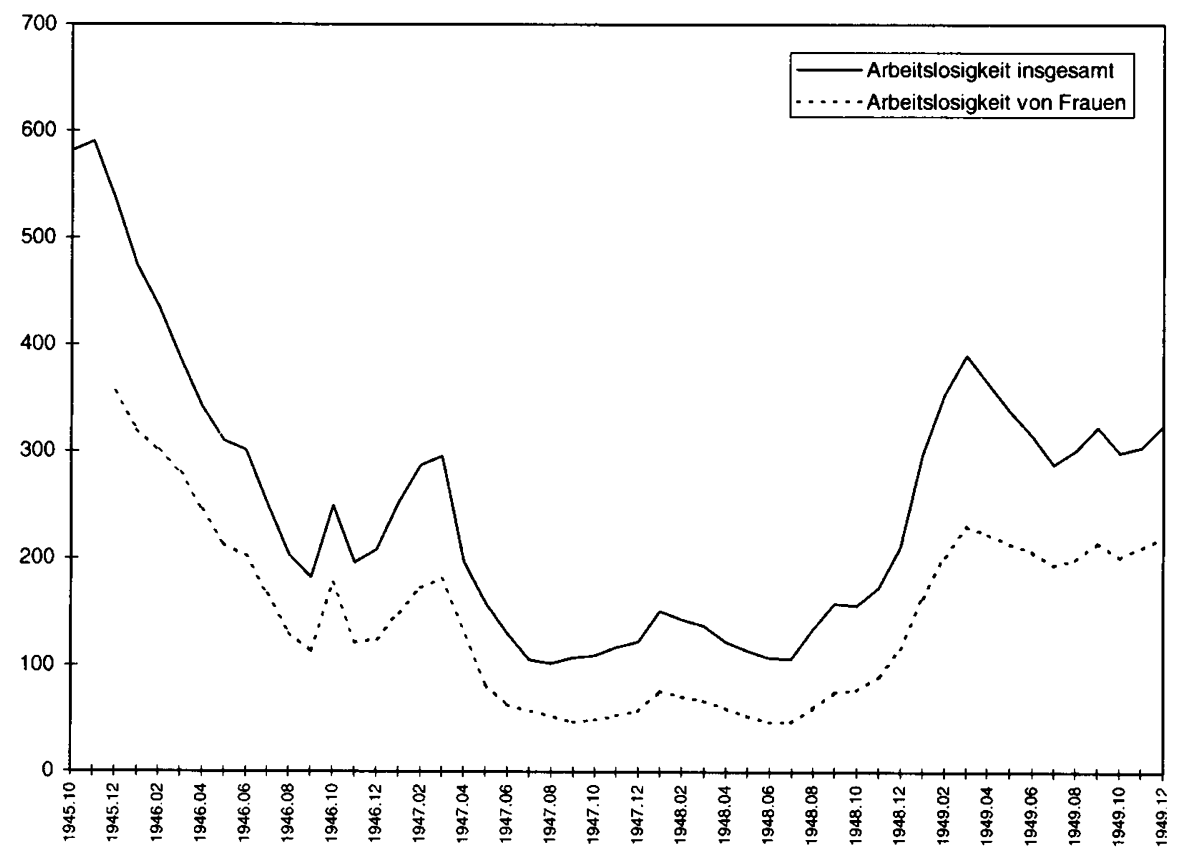

Quelle: Vgl. Tabelle 1 und 3 in diesem Band

darauf hingewiesen, daß mit dem Ansteigen der Arbeitslosigkeit auch ein leichter Anstieg der Erwerbstätigkeit und Beschäftigung zwischen Juni und Dezember 1948 verbunden war. Vgl. Buchheim, Die Währungsreform 1948 in Westdeutschland, S. 224.

135 Boldorf, Sozialfürsorge in der SBZ/DDR, S. 54.

136 Vgl. BAB, DQ 2/1919, Erläuterungen der HVAS (Abt. Statistik) vom 15. 3. 1949.

137 Vgl. zu den Zahlen ab 1950: Boldorf, Sozialfürsorge, S. 45; Zank, Wirtschaft und Arbeit, S. 173. 
Tabelle 1: Entwicklung der Arbeitslosigkeit in der SBZ (ohne Berlin) 1945-1949 (in Tausend)

\begin{tabular}{lccccc}
\hline Monat/Jahr & 1945 & 1946 & 1947 & 1948 & 1949 \\
\hline Januar & & 474 & 252 & 151 & 297 \\
Februar & & 435 & 287 & 143 & 354 \\
März & & 386 & 295 & 137 & 390 \\
April & & 342 & 197 & 122 & 364 \\
Mai & & 310 & 158 & 114 & 338 \\
Juni & & 301 & 130 & 107 & 315 \\
Juli & 251 & 105 & 106 & 288 \\
August & & 203 & 102 & 134 & 301 \\
September & & 182 & 107 & 158 & 323 \\
Oktober & 581 & 249 & 109 & 156 & 299 \\
November & 590 & 196 & 117 & 173 & 304 \\
Dezember & 536 & 208 & 122 & 211 & 325 \\
\hline
\end{tabular}

Quellen: Boldorf, Sozialfürsorge, S. 45. Eigene Angaben stammen aus: BAB, DQ 2/498, Bl. 247-250 [Dezember 1945]. Eine Addition der einzelnen Meldungen der Länder- bzw. Provinzverwaltungen ergibt dagegen für Dezember 1945 eine andere Summe: 595 487. BAB, DQ 2/2213, Statistische Übersichten über die Bevölkerungsstruktur und die Lage auf dem Arbeitsmarkt in der SBZ für Dezember 1945 , zusammengestellt von der ZVAS. BAB, DQ 2/112, Bl. 168, Vierteljahresbericht der DVAS vom 12. 5. 1947 [Januar-März 1947]. BAB, DQ 2/1949, Vierteljahresbericht der DVAS vom 7. 8. 1947 über die Arbeitsmarktlage [April-Juni 1947]. BAB, DQ 2/1949, Vierteljahresbericht der DVAS vom 12.11. 1947 über die Arbeitsmarktlage [Juli-September 1947]. BAB, DQ 2/817, Vierteljahresbericht der HVAS vom 27. 8. 1949 [Juni 1949]. BAB, DQ 2/817, Übersicht über die Arbeitsmarktstatistik der Länder der DDR [Oktober 1949].

Die prozentualen Angaben, d.h. der Anteil der Erwerbslosen an der Erwerbsbevölkerung, unterstreichen diese Entwicklung teilweise138: Im Januar 1946 lag der Anteil bei 7,2 Prozent, im Dezember desselben Jahres bei 2,7; auch hier lag der tiefste Stand im September 1947 (1,4 Prozent). Bis zum März 1949 kletterte diese Prozentzahl auf 4,9, im März 1950 lag sie bei 5,0 Prozent.

Die statistische Erfassung der Bevölkerung blieb im Untersuchungszeitraum keineswegs einheitlich, sondern unterlag zahlreichen Veränderungen, welche die ZVAS in enger Absprache mit der zuständigen SMAD-Abteilung durchführte. Karlshorst - das sei noch einmal betont - verfolgte sehr aufmerksam die Registrierung durch die Arbeitsverwaltung. Anfang 1946 verlangte die SMAD erneut „ernste Kontrollen der Arbeitsämter zwecks Erreichung einer zuverlässigen Statistik über die wirkliche Zahl der Arbeitslosen "139. Kurz darauf schlug der stellvertretende Chef der Abteilung Arbeitskraft Morenow ZVAS-Vertretern vor, Stichproben in „bedeutsamen Orten“ durchzuführen, um die statistischen Erhebungsmethoden überprüfen und verbessern zu können ${ }^{140}$. Ein schwerwiegender Eingriff erfolgte Anfang 1947: Seit März 1947 wurden die Arbeitspflichtigen, die keine Arbeit suchten, von den Arbeitslosen getrennt registriert. Durch diese statistische „Bereinigung“ sank die Gesamtgruppe der Erwerbslosen beträchtlich: Von

$138 \mathrm{Zu}$ den folgenden Zahlen: Zank, Wirtschaft und Arbeit, S. 173.

$139 \mathrm{BAB}, \mathrm{DQ} 2 / 1, \mathrm{Bl} .24$, Aktenvermerk über Besprechung mit der SMAD-Abt. Arbeitskraft am 4.1. 1946 (Hervorhebung im Original).

140 Ebenda, Bl. 31, Aktenvermerk über Besprechung am 23. 1. 1946. 
295257 (März 1947)141 auf 197477 (April 1947)142. Im Sommer 1948 regte die SMAD-Abteilung Arbeitskraft an, Teile der Arbeitsmarktstatistik - ähnlich wie in der Sowjetunion - vom Statistischen Zentralamt weiterführen zu lassen ${ }^{143}$. Die Vertreter der HVAS lehnten diesen Vorschlag mit der Begründung ab, daß die Arbeitsämter "mindestens" einmal monatlich statistische Unterlagen für die Lenkung der Arbeitskräfte erhalten sollten. Mit dieser Aufgabe sei das Statistische Zentralamt allerdings überfordert: So lägen die Ergebnisse der Berufszählung vom Oktober 1946 „bis jetzt" noch nicht vor ${ }^{144}$. Zur Stärkung der eigenen Position verwies der HVAS-Mitarbeiter Donau explizit auf entsprechende Bestimmungen des Kontrollratsbefehls Nr. 3 und der SMAD-Befehle Nr. 65 und 153, in denen die Arbeitsämter mit dieser Aufgabe beauftragt worden waren ${ }^{145}$. Kurz darauf begründete die Leitung der HVAS ihre Haltung ein weiteres Mal und konnte sich damit offensichtlich auch durchsetzen ${ }^{146}$.

Tabelle 2: Zabl der Arbeitspflichtigen in der SBZ (obne Berlin), die keine Arbeit suchen, 1947-1949 (in Tausend)

\begin{tabular}{lrcc}
\hline Monat/Jahr & 1947 & 1948 & 1949 \\
\hline Januar & & 292 & \\
Februar & 64 & 314 & 439 \\
März & 69 & 306 & \\
April & 156 & 317 & 437 \\
Mai & 173 & 311 & \\
Juni & 188 & 337 & 446 \\
Juli & 259 & 338 & \\
August & 278 & 352 & \\
September & 281 & 357 & 474 \\
Oktober & 290 & 364 & \\
November & 291 & 394 & \\
Dezember & & & \\
\hline
\end{tabular}

Quellen: BAB, DQ 2/1949, Vierteljahresbericht der DVAS vom 7. 8. 1947 [März-Juni 1947]. Die starke Zunahme dieses Personenkreises im Mai 1947 beruhte darauf, daß in den beiden Monaten zuvor nur geschätzte Zahlen vorlagen. BAB, DQ 2/1949, Vierteljahresbericht der DVAS vom 12.11. 1947 [Juli-September 1947]. BAB, DQ 2/1949, Vierteljahresbericht der DVAS vom 2. 3.1948 [OktoberDezember 1947]. BAB, DQ 2/179, Bl. 31, Bericht der HVAS vom 2. 4. 1948 [Januar 1948]. BAB, DQ 2/179, Bl. 48, Bericht der HVAS vom 4. 5. 1948 [Februar 1948]. BAB, DQ 2/3858, Bericht der HVAS vom 7. 6. 1948 [März 1948]. BAB, DQ 2/3858, Bericht der HVAS vom 8. 7.1948 [April 1948]. BAB, DQ 2/3403, Übersichten der HVAS [Mai, Juni, August-Dezember 1948]. SAPMO, DY 30/IV 2/2.027/ 21, Bl. 159 f., Vierteljahresbericht der HVAS vom 25. 5. 1949 [März 1949]. BAB, DQ 2/817, Vierteljahresbericht der HVAS vom 27. 8. 1949 [Juni 1949]. BAB, DQ 2/817, Vierteljahresbericht des Ministeriums für Arbeit und Gesundheitswesen vom 5. 12. 1949 [September 1949]. BAB, DQ 2/817, Vierteljahresbericht des Ministeriums für Arbeit und Gesundheitswesen vom 20. 3. 1950 [Dezember 1949].

$141 \mathrm{BAB}, \mathrm{DQ} 2 / 112$, Bl. 167-171, hier Bl. 168 (Rückseite), Vierteljahresbericht der DVAS vom 12. 5. 1947 über die Arbeitsmarktlage (Januar-März 1947).

${ }_{142}$ Ebenda, Bl. 174-179, hier Bl. 175 (Rückseite), Vierteljahresbericht der DVAS vom 7. 8. 1947 über die Arbeitsmarktlage (April-Juni 1947).

${ }_{143} \mathrm{BAB}, \mathrm{DQ} 2 / 2040$, Bl. 147, Aktennotiz über Besprechung bei der SMAD in Karlshorst am 29.6. 1948.

144 Ebenda.

${ }^{1+5}$ Ebenda, Bl. 143, Aktennotiz über Besprechung bei der SMAD am 1. 7. 1948.

$146 \mathrm{BAB}, \mathrm{DQ} 2 / 498$, Bl. 273 f., HVAS am 5. 7. 1948 an die SMAD-Abt. Arbeitskraft (Morenow). 
Insgesamt galt, daß die Arbeitsmarktlage bei DDR-Gründung für Männer günstiger war als für Frauen ${ }^{147}$. Das hing mit mehreren Faktoren zusammen: Von zentraler Bedeutung war die Rückkehr der allmählich aus der Kriegsgefangenschaft entlassenen Männer, die rasch zu einer Verdrängung der Frauen auf dem Arbeitsmarkt führte. Darüber hinaus berichteten zahlreiche Arbeitsämter schon Anfang 1946 über die "mangelnde Bereitwilligkeit vieler Betriebe, Frauen einzustellen" 148. Offensichtlich nutzten einige Betriebe auch die Umstellung von der Kriegs- auf die Friedenswirtschaft dazu, den Anteil der weiblich Beschäftigten zu reduzieren. In der Gruppe der Arbeitspllichtigen, die laut Statistik keine Arbeit suchten, befanden sich mehrheitlich weibliche Erwerbspflichtige: Darunter waren zahlreiche Hausfrauen, die unter die Registrierungspflicht fielen, die aber aufgrund der Belastung durch Kindererziehung und Haushaltsführung - oftmals als Alleinerziehende - eine Arbeit überhaupt nicht aufnehmen konnten. Insgesamt läßt sich feststellen, daß der Anstieg der Arbeitslosigkeit zur Jahreswende 1948/49 vor allem die weibliche Erwerbsbevölkerung traf. Deren Anteil an der Gesamterwerbslosenquote, die ohnehin stets über der der Männer lag, erhöhte sich nunmehr nochmals stärker. So erklärt sich auch der Umstand, daß unter den als arbeitslos registrierten Frauen die Gruppe der als vollarbeitsfähig Eingestuften dominierte. Das Verhältnis zu der Gruppe der erwerbsbeschränkten weiblichen Arbeitslosen lag im Laufe des Jahres 1949 ungefähr bei 5:1. Im Gegensatz dazu waren die männlichen Arbeitslosen mehrheitlich erwerbsbeschränkt eingestuft worden. Daraus zogen die SED-Führung sowie die Arbeitsverwaltung bei der Ausarbeitung des Zweijahrplanes die Konsequenz, daß zur Vergrößerung des Arbeitskräftepotentials in erster Linie die erwerbsfähigen Frauen zu mobilisieren waren. Diese Erkenntnis war nicht neu: Bereits Mitte Oktober 1947 hatte die DVAS die SMAD-Abteilung Arbeitskraft darauf hingewiesen, daß die „Arbeitskraftreserve bei den vollarbeitsfähigen Frauen im Alter von über 18 Jahren" größer sei als bei den Männern ${ }^{149}$, bei denen die Berliner Zentralverwaltung eine Langzeitarbeitslosigkeit nicht mehr feststellen konnte.

Einen großen Anteil in der Gruppe der erwerbsbeschränkten Arbeitslosen hatten die Flüchtlinge und Vertriebenen, die ebenfalls der Registrierungspflicht unterlagen und aufgrund der Vertreibung gesundheitlich stark angeschlagen waren. Es deutet einiges darauf hin, daß unter den "Umsiedlern“ der Anteil der als arbeitsfähig eingestuften Personen erheblich unter dem prozentualen Anteil bei der eingesessenen Bevölkerung lag150. Das Ansteigen der Erwerbs-

${ }^{147}$ So auch das Urteil bei: Zank, Wirtschaft und Arbeit, S. 173.

148 BAB, DQ 2/2213, Bericht der ZVAS (Abt. II a) vom 6. 3. 1946 über die Arbeitsmarktlage in Sachsen-Anhalt im Januar 1946.

149 BAB, DQ 2/512, Abt. I a am 17. 10. 1947 an SMAD (Morenow).

150 Vgl. Hoffmann, Vertriebenenintegration durch Arbeitsmarktlenkung?, S. 179. Im Frühjahr 1949 stellte die HVAS die gesonderte Erfassung der "Umsiedler“ ganz ein. Begründet wurde dieser Schritt mit einer angeblichen Vereinfachung des statistischen Meldewesens; entscheidend dürfte aber vielmehr die Überzeugung gewesen sein, daß die Einglicderung der Flüchtlinge und Vertriebenen vor dem vermeintlich erfolgreichen Abschluß stand und deshalb eine besondere Betreuung diescr Bevölkerungsgruppe nicht mehr erforderlich schien. Letztlich waren also politische Überlegungen von ausschlaggebender Bedeutung. Vgl. BAB, DQ 2/817, Vierteljahresbericht der HVAS über die Arbcitsmarktlage in der SBZ (April-Juni 1949), S. 8. 
Tabelle 3: Arbeitslosigkeit von Frauen in der SBZ (obne Berlin) 1945-1949 (in Tausend)

\begin{tabular}{|c|c|c|c|c|c|}
\hline Monat/Jahr & 1945 & 1946 & 1947 & 1948 & 1949 \\
\hline Januar & & 320 & 149 & 76 & 164 \\
\hline Februar & & 300 & 172 & 71 & 202 \\
\hline März & & 279 & 181 & 67 & 230 \\
\hline April & & 245 & & 60 & 223 \\
\hline Mai & & 213 & 82 & 53 & 214 \\
\hline Juni & & 202 & 63 & 47 & 207 \\
\hline Juli & & 166 & & 47 & 194 \\
\hline August & & 130 & & 60 & 199 \\
\hline September & & 113 & 47 & 75 & 215 \\
\hline Oktober & & 177 & 49 & 77 & 201 \\
\hline November & & 121 & 53 & 90 & \\
\hline Dezember & 355 & 124 & 57 & 116 & 220 \\
\hline
\end{tabular}

Quellen: BAB, DQ 2/498, Bl. 247-250, hier Bl. 250 [Dezember 1945]. BAB, DQ 2/1949, Vierteljahresbericht der DVAS vom 8.6. 1946 über die Arbeitsmarktlage [Januar-März 1946]. BAB, DQ 2/ 1949, Vierteljahresbericht der DVAS vom 1.8. 1946 über die Arbeitsmarktlage [April-Juni 1946]. BAB, DQ 2/3403, Bl. 258, Übersicht der DVAS [Juli-November 1946]. BAB, DQ 2/1949, Vierteljahresbericht der DVAS vom 28. 1. 1947 über die Arbeitsmarktlage [Oktober-Dezember 1946]. BAB, DQ 2/112, Bl. 167-171, hier Bl. 168, Vierteljahresbericht der DVAS vom 12. 5.1947 über die Arbeitsmarktlage [Dezember 1946-März 1947]. SAPMO, NY 4182/1158, Bl. 158-163, hier Bl. 159, Bericht der DVAS vom 2. 7. 1947 über die Arbeitsmarktlage [Mai 1947]. BAB, DQ 2/112, Bl. 174-179, hier Bl. 176, Vierteljahresbericht der DVAS vom 7. 8. 1947 über die Arbeitsmarktlage [Juni 1947]. BAB, DQ 2/1949, Vierteljahresbericht der DVAS vom 12.11. 1947 über die Arbeitsmarktlage [September 1947]. BAB, DQ 2/3858, Übersicht der DVAS [Oktober 1947]. BAB, DQ 2/3858, Übersicht der DVAS [November 1947]. BAB, DQ 2/1949, Vierteljahresbericht der DVAS vom 2.3. 1948 über die Arbeitsmarktlage [Dezember 1947]. BAB, DQ 2/3858, Bericht der HVAS vom 2. 4. 1948 über die Arbeitsmarktlage [Januar 1948]. BAB, DQ 2/3858, Bericht der HVAS vom 4. 5. 1948 über die Arbeitsmarktlage [Februar 1948]. BAB, DQ 2/3858, Vierteljahresbericht der HVAS vom 7.6. 1948 [März 1948]. BAB, DQ 2/3858, Bericht der HVAS vom 8. 7.1948 über die Arbeitsmarktlage [April 1948]. $\mathrm{BAB}, \mathrm{DQ} 2 / 3403, \mathrm{Bl}$. 1, vorläufige Zonenübersicht der HVAS [Mai 1948]. BAB, DQ 2/3403, Bl. 3, vorläufige Übersicht der HVAS [Juni 1948]. BAB, DQ 2/3403, Bl. 12, vorläufige Übersicht der HVAS [Juli 1948]. BAB, DQ 2/3403, Bl. 14, vorläufige Übersicht der HVAS [August 1948]. BAB, DQ $2 /$ 3403, Bl. 16, vorläufige Übersicht der HVAS [September 1948]. BAB, DQ 2/3403, Bl. 19, Übersicht der HVAS [Oktober 1948]. BAB, DQ 2/3403, Bl. 20, Übersicht der HVAS [November 1948]. BAB, DQ 2/3403, Übersicht der HVAS [Dezember 1948]. BAB, DQ 2/3403, Bl. 25, Übersicht der HVAS [Januar 1949]. BAB, DQ 2/3403, Bl. 28, Übersicht der HVAS [Februar 1949]. BAB, DQ 2/3403, Bl. 36, Übersicht der HVAS [März 1949]. BAB, DQ 2/3403, Bl. 40, vorläufige Übersicht der HVAS [April 1949]. BAB, DQ 2/3867, vorläufige Übersicht der HVAS [Mai 1949]. BAB, DQ 2/3867, Übersicht der HVAS [Juni 1949]. BAB, DQ 2/3867, Übersicht der HVAS [Juli 1949]. BAB, DQ 2/2070, Erläuterungen der HVAS (Abt. Statistik) vom 21.9. 1949 [August 1949]. BAB, DQ 2/817, Vierteljahresbericht des Ministeriums für Arbeit und Gesundheitswesen vom 5.12. 1949 [September 1949]. $\mathrm{BAB}, \mathrm{DQ} 2 / 817$, Übersicht des Ministeriums für Arbeit und Gesundheitswesen [Oktober 1949]. BAB, DQ 2/2070, Statistische Übersicht des Ministeriums für Arbeit und Gesundheitswesen über die Arbeitslage [Dezember 1949]. 
Tabelle 4: Zabl der erwerbsbeschränkten Arbeitslosen in der SBZ (obne Berlin) 1946-1949 (in Tausend)

\begin{tabular}{lcccc}
\hline Monat/Jahr & 1946 & 1947 & 1948 & 1949 \\
\hline Januar & & & 88 & 113 \\
Februar & & & 84 & 124 \\
März & 209 & 143 & 81 & 131 \\
April & 238 & 105 & 77 & 126 \\
Mai & 189 & 88 & 70 & 119 \\
Juni & 176 & 82 & 66 & 116 \\
Juli & 156 & 72 & 65 & 107 \\
August & 132 & 70 & 70 & 102 \\
September & 115 & 71 & 79 & 100 \\
Oktober & 110 & & 77 & 95 \\
November & 116 & & 80 & 95 \\
Dezember & 118 & 80 & 93 & 100 \\
\hline
\end{tabular}

Quellen: BAB, DQ 2/1949, Vierteljahresbericht der ZVAS vom 8. 6. 1946 [März 1946]. BAB, DQ 2/ 1949, Vierteljahresbericht der DVAS vom 1. 8. 1946 [April-Juni 1946]. BAB, DQ 2/1949, Vierteljahresbericht der DVAS [Juli-September 1946]. BAB, DQ 2/1949, Vierteljahresbericht der DVAS vom 28. 1. 1947 [Oktober-Dezember 1946]. BAB, DQ 2/1949, Vierteljahresbericht der DVAS vom 12. 5. 1947 [März 1947]. BAB, DQ 2/1949, Vierteljahresbericht der DVAS vom 7. 8. 1947 [April-Juni 1947]. BAB, DQ 2/1949, Vierteljahresbericht der DVAS vom 12.11. 1947 [Juli-September 1947]. BAB, DQ 2/1949, Vierteljahresbericht der DVAS vom 2.3. 1948 [Dezember 1947]. BAB, DQ 2/179, Bl. 31, Bericht der HVAS vom 2. 4. 1948 [Januar 1948]. BAB, DQ 2/179, Bl. 48, Bericht der HVAS vom 4. 5. 1948 [Februar 1948]. BAB, DQ 2/179, BI. 1, Ubersicht der HVAS [März 1948]. BAB, DQ 2/3858, Bericht der HVAS vom 8. 7.1948 [April 1948]. BAB, DQ 2/3858, Bericht der HVAS vom 7. 7.1948 [Mai 1948]. BAB, DQ 2/3403, Übersichten der HVAS [Juni, August, September, Dezember 1948]. BAB, DQ 2/970, HVAS am 2. 3. 1949 an SMAD-Abt. Arbeitskraft [Juli 1948, Januar 1949]. BAB, DQ 2/ 4048, Übersicht der HVAS [Februar 1949]. SAPMO, DY 30/IV 2/2.027/21, Bl. 159, Vierteljahresbericht der HVAS vom 25. 5. 1949 [März 1949]. BAB, DQ 2/4048, Übersicht der HVAS [April 1949]. $\mathrm{BAB}, \mathrm{DQ} 2 / 4048$, Übersicht der HVAS [Mai 1949]. BAB, DQ 2/817, Vierteljahresbericht der HVAS vom 27. 8. 1949 [Juni 1949]. BAB, DQ 2/4048, Übersicht der HVAS [Juli 1949]. BAB, DQ 2/2070, Erläuterungen der HVAS vom 21. 9. 1949 [August 1949]. BAB, DQ 2/817, Vierteljahresbericht des Ministeriums für Arbeit und Gesundheitswesen vom 5. 12. 1949 [September 1949]. BAB, DQ 2/817, Übersicht des Ministeriums für Arbeit und Gesundheitswesen [Oktober 1949]. BAB, DQ 2/3403 Übersichten der HVAS [Oktober, November 1949]. BAB, DQ 2/1013, Bericht des Ministeriums für Arbeit und Gesundheitswesen vom 9.1. 1950 [November 1949]. BAB, DQ 2/817, Vierteljahresbericht des Ministeriums für Arbeit und Gesundheitswesen vom 20. 3. 1950 [Dezember 1949].

beschränkten unter den Arbeitslosen führte auch die DVAS auf die „Umsiedler“ zurück, ,von denen nur ein geringer Prozentsatz volleinsatzfähig“ sei151. Als weiterer belastender Faktor für den Arbeitsmarkt wurden die Kriegsheimkehrer genannt. 
Tabelle 5: Zabl der offenen Stellen in der SBZ (ohne Berlin) 1946-1949 (in Tausend)

\begin{tabular}{lcrrr}
\hline Monat/Jahr & 1946 & 1947 & 1948 & 1949 \\
\hline Januar & 156 & 92 & 140 & 53 \\
Februar & 180 & 89 & 157 & 52 \\
März & 192 & 100 & 169 & 59 \\
April & 157 & & 186 & 64 \\
Mai & 147 & 166 & 185 & 70 \\
Juni & 144 & 187 & 189 & 77 \\
Juli & & 201 & 193 & 100 \\
August & & 206 & 168 & 95 \\
September & 199 & 155 & 93 \\
Oktober & 148 & 196 & 142 & 88 \\
November & 121 & 171 & 111 & 67 \\
Dezember & 105 & 141 & 72 & 53 \\
\hline
\end{tabular}

Quellen: BAB, DQ 2/1949, Vierteljahresbericht der ZVAS vom 8.6. 1946 [Januar-März 1946]. BAB, DQ 2/1949, Vierteljahresbericht der DVAS vom 1. 8. 1946 [April-Juni 1946]. BAB, DQ 2/1949, Vierteljahresbericht der DVAS vom 28.1.1947 [Oktober-Dezember 1946]. BAB, DQ 2/1949, Vierteljahresbericht der DVAS vom 12. 5. 1947 [Januar-März 1947]. SAPMO, NY 4182/1158, Bl. 158-163, hier Bl. 159, Bericht der DVAS vom 2. 7. 1947 über dic Arbeitsmarktlage [Mai 1947]; BAB, DQ 2/1949, Vierteljahresbericht der DVAS vom 12.11. 1947 [Juni-September 1947]. BAB, DQ 2/1949, Vierteljahresbericht der DVAS vom 2. 3. 1948 [Oktober-Dezember 1947]. BAB, DQ 2/179, Bl. 31, Bericht der HVAS vom 2. 4. 1948 [Januar 1948]. BAB, DQ 2/179, Bl. 48, Bericht der HVAS vom 4. 5. 1948 [Februar 1948]. BAB, DQ 2/3858, Bericht der HVAS vom 7. 6. 1948 [März 1948]. BAB, DQ 2/3858, Bericht der HVAS vom 8. 7. 1948 [April 1948]. BAB, DQ 2/3858, Bericht der HVAS vom 7. 7.1948 [Mai 1948]. BAB, DQ 2/3403, Übersichten der HVAS [Juni-August 1948]. SAPMO, DY 34/21434 [September-Dezember 1948]. BAB, DQ 2/4048, Übersicht der HVAS [Januar 1949]. BAB, DQ 2/ 4048, Übersicht der HVAS [Februar 1949]. SAPMO, DY 30/IV 2/2.027/21, Bl. 159, Vierteljahresbericht der HVAS vom 25. 5. 1949 [März 1949]. BAB, DQ 2/4048, Übersicht der HVAS [April 1949]. $\mathrm{BAB}, \mathrm{DQ} 2 / 4048$, Übersicht der HVAS [Mai 1949]. BAB, DQ 2/817, Vierteljahresbericht der HVAS vom 27. 8. 1949 [Juni 1949]. BAB, DQ 2/4048, Übersicht der HVAS [Juli 1949]. BAB, DQ 2/3867, Übersicht der HVAS [August 1949]. BAB, DQ 2/4048, Übersicht der HVAS [September 1949]. BAB, DQ 2/817, Übersicht des Ministeriums für Arbeit und Gesundheitswesen [Oktober 1949]. BAB, DQ 2/1013, Bericht des Ministeriums für Arbeit und Gesundheitswesen vom 9. 1. 1950 [November 1949]. $\mathrm{BAB}, \mathrm{DQ} 2 / 817$, Vierteljahresbericht des Ministeriums für Arbeit und Gesundheitswesen vom 20.3. 1950 [Dezember 1949].

Die vergleichsweise hohe Zahl der offenen Stellen in der SBZ bis zum Jahresende 1948 erklärt sich vor allem aus den sowjetischen Arbeitskräfteanforderungen. Von den Arbeitsämtern wurden die Forderungen der sowjetischen Besatzungsmacht oftmals als offene Stellen ausgewiesen. In ihren Quartalsberichten wies die DVAS selbst auf diesen Zusammenhang hin ${ }^{152}$. Die Deutsche Zentralverwaltung für Industrie befürchtete ihrerseits ein Ansteigen der Arbeitslosenzahlen, sobald die Demontagearbeiten abgeschlossen waren ${ }^{153}$. Dabei ist jedoch zu berücksichtigen, daß die Demontagen nicht nur positive Beschäftigungsauswirkungen hatten, son-

152 Vgl. ebenda, Vierteljahresbericht der DVAS vom 1. 8. 1946 über die Arbeitsmarktlage in der SBZ (April-Juni 1946), S. 5.

153 BAB, DQ 2/2060, Protokoll über die Besprechung der Vertreter der Zentralverwaltungen am 7. 5. 1946, S. 2. 
dern auch zu nicht unerheblichen Störungen der betrieblichen Produktion geführt haben, da Arbeitskräfte zum Teil aus der laufenden Produktion herausgezogen und sowjetischen Demontagebetrieben zugeführt bzw. entlassen wurden, weil es die Betriebe nach der Demontage faktisch nicht mehr gab.

Auffallend ist der dramatische Rückgang der gemeldeten offenen Stellen ab Dezember 1948, während die Arbeitsämter nahezu zeitgleich ein Ansteigen der Arbeitslosenzahlen registrierten. Zwischen beiden Entwicklungen bestand daher spiegelbildlich eine negative Korrelation, wie die Abteilung Statistik der HVAS Ende Januar 1949 treffend feststellte ${ }^{154}$. Die Hauptverwaltung wies darauf hin, daß die Zahl der offenen Stellen Ende Dezember 1948 erstmals unter der Gesamtzahl der männlichen Arbeitslosen gelegen hatte. Das verdeutlichte zum einen den oben beschriebenen Rückgang der sowjetischen Arbeitskräfteanforderungen, zum anderen die immer geringer werdende Bereitschaft oder Fähigkeit ostdeutscher Betriebe, neue Arbeitskräfte einzustellen.

Sowohl die HVAS als auch die Landesverwaltungen stellten die Ende 1948 einsetzende Entlassungswelle als „Beginn eines Gesundungsprozesses“ der SBZWirtschaft dar ${ }^{155}$. So erklärte der sächsische Minister für Industrie und Verkehr, Gerhart Ziller, auf einer Arbeitstagung am 10. Februar 1949, daß die Betriebe die Vorgabe erhalten hätten, die Arbeitsproduktivität zu erhöhen. Daher seien auch zahlreiche "gehortete Arbeitskräfte" vor allem in der Textilindustrie entlassen worden ${ }^{156}$. Damit wurde ein Phänomen angesprochen, das im Verlauf der fünfziger Jahre an Bedeutung gewinnen sollte: Aufgrund der zentralen Planungsvorgaben gingen immer mehr Betriebe dazu über, knappe Ressourcen zu horten, um für wirtschaftlich schlechte Zeiten gewappnet zu sein. Auch die HVAS-Leitung betonte gegenüber der Abteilung Arbeitskraft der SMAD in Karlshorst, daß sich die Steigerung der Arbeitsproduktivität „arbeitskräftesparend“ auswirke ${ }^{157}$. Einzelne Landesarbeitsämter wandten sich an die Berliner Hauptverwaltung mit der Bitte, Kredite für die betroffenen Gemeinden durch die DWK zu bewilligen, um „für die Übergangszeit Straßenbauten und Enttrümmerungsarbeiten" durchzuführen 158 . Die Arbeitsverwaltung erkannte diese sich verschlechternde Entwicklung und forderte daraufhin die SED-Führung auf, gezielt Investitionsprogramme zur Bereitstellung neuer Arbeitsplätze vorzubereiten.

$154 \mathrm{BAB}, \mathrm{DQ} 2 / 970$, Bericht der HVAS (Abt. Statistik) vom 25. 1. 1949, S. 1.

155 So der sächsische Wirtschaftsminister Ziller am 10.2. 1949. BAB, DQ 2/2040, Bl. 186-188, hier Bl. 187.

156 Ebenda.

$157 \mathrm{BAB}, \mathrm{DQ}$ 2/970, HVAS am 2. 3. 1949 an SMAD-Abt. Arbeitskraft (Morenow), S. 3.

158 Ebenda, Aktenvermerk der HVAS-Abt. I a vom 31. 1. 1949. 